\title{
Aging and high-fat diet feeding lead to peripheral insulin resistance and sex- dependent changes in brain of mouse model of tau pathology THY-Tau22
}

Miroslava Kacírová1, Blanka Železná ${ }^{1}$, Michaela Blažková1, Martina Holubová1, Andrea Popelová1, Jaroslav Kuneš1,2, Blanka Šedivá ${ }^{3}$ and Lenka Maletínská ${ }^{* *}$

\begin{abstract}
Background: Obesity leads to low-grade inflammation in the adipose tissue and liver and neuroinflammation in the brain. Obesity-induced insulin resistance (IR) and neuroinflammation seem to intensify neurodegeneration including Alzheimer's disease. In this study, the impact of high-fat (HF) diet-induced obesity on potential neuroinflammation and peripheral IR was tested separately in males and females of THY-Tau22 mice, a model of tau pathology expressing mutated human tau protein.

Methods: Three-, 7-, and 11-month-old THY-Tau22 and wild-type males and females were tested for mobility, anxiety-like behavior, and short-term spatial memory in open-field and Y-maze tests. Plasma insulin, free fatty acid, cholesterol, and leptin were evaluated with commercial assays. Liver was stained with hematoxylin and eosin for histology. Brain sections were 3',3'-diaminobenzidine (DAB) and/or fluorescently detected for ionized calciumbinding adapter molecule 1 (Iba1), glial fibrillary acidic protein (GFAP), and tau phosphorylated at T231 (pTau (T231)), and analyzed. Insulin signaling cascade, pTau, extracellular signal-regulated kinase 1/2 (ERK1/2), and protein phosphatase 2A (PP2A) were quantified by western blotting of hippocampi of 11-month-old mice. Data are mean \pm SEM and were subjected to Mann-Whitney t test within age and sex and mixed-effects analysis and Bonferroni's post hoc test for age comparison.

Results: Increased age most potently decreased mobility and increased anxiety in all mice. THY-Tau22 males showed impaired short-term spatial memory. HF diet increased body, fat, and liver weights and peripheral IR. HF diet-fed THY-Tau22 males showed massive Iba1+ microgliosis and GFAP+ astrocytosis in the hippocampus and amygdala. Activated astrocytes colocalized with pTau (T231) in THY-Tau22, although no significant difference in hippocampal tau phosphorylation was observed between 11-month-old HF and standard diet-fed THY-Tau22 mice. Eleven-month-old THY-Tau22 females, but not males, on both diets showed decreased synaptic and postsynaptic plasticity.
\end{abstract}

\footnotetext{
*Correspondence: lenka.maletinska@uochb.cas.cz

${ }^{1}$ Institute of Organic Chemistry and Biochemistry of the Czech Academy of Sciences, Flemingovo náměstí 542/2, 16000 Prague 6, Czech Republic

Full list of author information is available at the end of the article
}

(c) The Author(s). 2021 Open Access This article is licensed under a Creative Commons Attribution 4.0 International License, which permits use, sharing, adaptation, distribution and reproduction in any medium or format, as long as you give appropriate credit to the original author(s) and the source, provide a link to the Creative Commons licence, and indicate if changes were made. The images or other third party material in this article are included in the article's Creative Commons licence, unless indicated otherwise in a credit line to the material. If material is not included in the article's Creative Commons licence and your intended use is not permitted by statutory regulation or exceeds the permitted use, you will need to obtain permission directly from the copyright holder. To view a copy of this licence, visit http://creativecommons.org/licenses/by/4.0/ The Creative Commons Public Domain Dedication waiver (http://creativecommons.org/publicdomain/zero/1.0/) applies to the data made available in this article, unless otherwise stated in a credit line to the data. 
Conclusions: Significant sex differences in neurodegenerative signs were found in THY-Tau22. Impaired short-term spatial memory was observed in 11-month-old THY-tau22 males but not females, which corresponded to increased neuroinflammation colocalized with pTau(T231) in the hippocampi and amygdalae of THY-Tau22 males. A robust decrease in synaptic and postsynaptic plasticity was observed in 11-month-old females but not males. HF diet caused peripheral but not central IR in mice of both sexes.

Keywords: Alzheimer's disease, THY-Tau22 mouse, Obesity, Neuroinflammation, Peripheral insulin resistance, Sex differences

\section{Introduction}

The prevalence of obesity is increasing worldwide [1], leading to diverse comorbidities and increasing the cost of their cure. Obesity can cause insulin resistance (IR) not only in the periphery but also in the brain. IR is characterized by reduced sensitivity of insulinotropic tissues to the action of insulin and is one of the major pathophysiological attributes of type 2 diabetes mellitus [2]. In addition, obesity supports low-grade inflammation in the adipose tissue and the liver and neuroinflammation in the hypothalamus [2, 3]. Recently, obesityrelated neuroinflammation has been detected in extrahypothalamic brain structures such as the hippocampus or brain cortex (reviewed by [3, 4]) and linked to impaired cognitive function. Both IR and low-grade neuroinflammation resulting from obesity seem to intensify neurodegenerative changes in brains affected by Alzheimer's disease $(\mathrm{AD})$ [5].

In addition to beta-amyloid (A $\beta)$ plaques, hyper and abnormally phosphorylated tau protein [6], which is prone to form neurofibrillary tangles and finally to cause synapse failure, is another main AD hallmark. The transgenic model THY-Tau22 was established to elucidate the role of tau pathology and related pathological changes in the brains of patients with AD and other tauopathies [7]. THY-Tau22 mice overexpress human four-repeat tau with G272V and P301S mutations under the Thy1.2 promoter, mainly in the brain, and therefore are nearly free of motor dysfunctions. These mice develop tau hyper and abnormal phosphorylation, neurofibrillary tangles, loss of functional synapses, astrogliosis, and mild cognitive impairment, progressing with age [7-9].

In THY-Tau22 mice, a potential interrelation between tau pathology and astroglial and microglial immune responses was shown and linked to an early chemokine response that attracted $\mathrm{T}$ cells into the hippocampus [10]. Furthermore, the increasing presence of hippocampal CD11b (integrin $\alpha M$ ) with increasing age was observed in the THY-Tau22 mouse model. In addition, the mRNA expression of innate immunity markers Tlr2 (Toll-like receptor 2), CD68 (cluster of differentiation 68), and TNF $\alpha$ (tumor necrosis factor $\alpha$ ) was found to be increased [10]. This finding points to a fundamental role of neuroinflammation in tau pathology.
Neuroinflammation, represented by an increase in reactive glial cells and cytokine activation, was also found in the hypothalami of insulin-deficient rodents [11] and those with high-fat (HF) diet-induced obesity and in obese humans [12]. HF diet-induced obesity in rodents was reported to cause IR that mostly cohered with enhanced tau phosphorylation [13, 14], mainly in the hippocampus. However, others found neither tau hyperphosphorylation nor neuroinflammation in the hippocampi and brain cortex of male Wistar rats fed a high-fat/high-sucrose diet [15]. Moreover, HF dietinduced obesity-but neither peripheral nor central IR-was specified as the reason for hippocampal tau hyperphosphorylation and worsened learning ability in THY-Tau22 mice; however, no link of HF dietinduced obesity to neuroinflammation was mentioned in the study [16]. Furthermore, Mangold et al. found sexually divergent neuroinflammation with aging in C57BL6 mice that might contribute to sex differences in age-related neurological diseases such as stroke and Alzheimer's disease, specifically in the complement system. They found that age-related hippocampal induction of neuroinflammatory gene expression was sexually divergent and enriched for microgliaspecific genes such as complement pathway components. It seems that age is the major cause of difference in neuroinflammation between sexes [17].

The present study aims to determine if age-dependent obesity resulting from HF diet feeding causes IR in the periphery, affects insulin signaling in the hippocampus, leads to impaired age-related behavioral and memory changes, and worsens neuroinflammation in the brain separately in males and females of THY-Tau 22 mice and corresponding wild-type (wt) controls. Additionally, we examine whether these two pathologies-potential neuroinflammation resulting from obesity and pathological tau-colocalize in brains of THY-Tau22 mice and corresponding wt controls.

\section{Materials and methods \\ Animals}

THY-Tau22 male and female mice with a C57Bl/6 J background, generous gift of Dr. Luc Buée (Lille Neuroscience \& Cognition, Inserm UMR1172, France), were 
genotyped [7] and bred at the Biotechnology and Biomedicine Centre of the Academy of Sciences and Charles University (BIOCEV) (Vestec, Czech Republic). From the age of 7 weeks, the mice were housed and handled in the animal facility of the Institute of Organic Chemistry and Biochemistry of the Czech Academy of Sciences, Prague, Czech Republic, with a 12-h light/dark cycle (lights on at 6 a.m.) and temperature set at $23 \pm$ $2{ }^{\circ} \mathrm{C}$. Wt C57Bl $/ 6 \mathrm{~J}$ mice of both sexes were used as controls. Mice were housed 4-6 per cage with ad libitum access to water and food (Table 1). The wt and THYTau22 groups of both sexes sacrificed at 3 months $(\mathrm{n}=$ 4-5) were fed a standard (St) diet (standard rodent diet Ssniff $^{\circ}$ R/M-H (Ssniff Spezialdiäten GmbH, Soest, Germany)). The wt and THY-Tau22 mice of both sexes sacrificed at $7(n=7-8)$ or $11(n=7-14)$ months of age were fed either a St or HF diet. Feeding the HF diet started from 8 weeks of age. The caloric content percentage values for the HF diet were 13\% proteins, $60 \%$ fats, and 27\% carbohydrates [18]. All mice were weighed once per week when the HF diet feeding started until the day of dissection. The age of mice sacrification was designed based on the previous model characterization where the particular signs of neurodegeneration were established [7, 9].

The animal experiments followed the ethical guidelines for animal experiments in the Czech Republic Act Nr. 246/1992 and were approved by the Committee for Experiments with Laboratory Animals of the Czech Academy of Sciences.

\section{Behavioral tests}

Behavioral tests were performed 2 weeks before the dissection (Table 1).

\section{Open field}

Anxiety-like behavior, exploration, and locomotion were tested by the open field behavioral test [19], which was performed in a $50 \times 50 \times 50 \mathrm{~cm}^{3}$ box. The mouse was placed into the middle part of the box, and the recording started immediately after placement and lasted for 10 min. The obtained record was analyzed using EthoVision
XT software (Noldus, Wageningen, Netherlands). The average velocity and wall distance were monitored. The average velocity includes the mouse behavior including grooming and freezing.

\section{Y-maze}

Short-term spatial memory was tested by a Y-maze behavioral test described previously [20]. The obtained records were analyzed using EthoVision XT software.

The average time spent in the new zone (NZ) and the average number of entries into the NZ at the second session of the experiment were monitored.

\section{Oral glucose tolerance test}

One week before behavioral tests (Table 1), 7- and 11month-old mice were tested for tolerance to glucose with an oral glucose tolerance test (OGTT). After $6 \mathrm{~h}$ of fasting, the concentration of blood glucose was measured in the blood from the tip of the tail using a Glucocard $^{\mathrm{TM}} \mathrm{X}$-meter (Arkray factory, Inc., Japan) as described previously [21]. Briefly, glucose at a dose of 2 $\mathrm{g} / \mathrm{kg}$ of mouse body weight was administered by gavage. Blood glucose concentration was determined at 15, 30, $60,90,120$, and $180 \mathrm{~min}$ after glucose gavage.

The time curves of $\Delta$ Glucose were plotted; $\Delta$ Glucose $=\mathrm{c}_{\mathrm{Glc}}(\mathrm{t})-\mathrm{c}_{\mathrm{Glc}}\left(\mathrm{t}_{0}\right)$, where $\mathrm{c}_{\mathrm{Glc}}(\mathrm{t})$ is the concentration of glucose at a particular time $t$ and $c_{G l c}\left(t_{0}\right)$ is the concentration of glucose at time $0 \mathrm{~min}$. The areas under the curves $\left(\mathrm{AUC}_{\Delta \text { glucose }}\right)$ were calculated using GraphPad Prism 8.

\section{Blood plasma inflammation marker}

The concentration of C-reactive protein (CRP) in nonanesthetized fasting plasma was determined using Mouse CRP ELISA kit (Thermo Scientific, Frederick, MD, USA). The CRP was determined in 11-month-old mice of both sexes. The measurement was performed according to manufacturer's instructions.

\section{Organ dissection}

Blood samples from the overnight fasád animals were collected from the tail veins, and the concentrations of

Table 1 Experimental study design time course of THY-Tau22 and wt

\begin{tabular}{|c|c|c|c|c|c|c|c|c|c|c|}
\hline \multirow{2}{*}{$\begin{array}{l}\text { Age } \\
\text { Weeks on diet }\end{array}$} & \multirow[b]{2}{*}{0} & \multirow[b]{2}{*}{1} & \multicolumn{2}{|l|}{3 months } & \multicolumn{3}{|l|}{7 months } & \multicolumn{3}{|l|}{11 months } \\
\hline & & & 6 & 8 & 22 & 23 & 24 & 37 & 38 & 42 \\
\hline Diet, treatment & St & St & $\begin{array}{l}\text { Behavioral } \\
\text { tests }\end{array}$ & $\begin{array}{l}\text { Dissection after } \\
12 \mathrm{~h} \text { fasting }\end{array}$ & - & - & - & - & - & - \\
\hline Diet, treatment & St & $\mathrm{St} / \mathrm{HF}$ & - & - & $\begin{array}{l}\text { OGTT after } \\
6 \mathrm{~h} \text { fasting }\end{array}$ & $\begin{array}{l}\text { Behavioral } \\
\text { tests }\end{array}$ & $\begin{array}{l}\text { Dissection after } \\
12 \mathrm{~h} \text { fasting }\end{array}$ & - & - & - \\
\hline Diet, treatment & St & $\mathrm{St} / \mathrm{HF}$ & - & - & - & - & - & $\begin{array}{l}\text { OGTT after } \\
6 \text { h fasting }\end{array}$ & $\begin{array}{l}\text { Behavioral } \\
\text { tests }\end{array}$ & $\begin{array}{l}\text { Dissection after } \\
12 \mathrm{~h} \text { fasting }\end{array}$ \\
\hline
\end{tabular}

Week 0 on diet corresponds to 7 weeks of age 
blood glucose were measured. The blood plasma was separated with EDTA and stored at $-80^{\circ} \mathrm{C}$. Then, the mice were deeply anesthetized with pentobarbital (170 $\mathrm{mg} / \mathrm{kg}$ of mouse body weight, Sigma Aldrich, St. Louis, MO, USA), the left heart ventricle was punctured, blood was collected, and EDTA plasma was isolated and stored at $-80^{\circ} \mathrm{C}$. The mice were transcardially perfused with saline solution supplemented with heparin $(10 \mathrm{U} / \mathrm{ml}$, Zentiva, Prague, Czech Republic).

Brains were dissected on ice. The right hemispheres of each brain were postfixed in $4 \%$ paraformaldehyde (PFA) as described previously [22] and stored in a $30 \%$ sucrose solution with the addition of sodium azide until cutting for immunohistochemistry (IHC). The hippocampi from the left hemispheres were covered with $200 \mu \mathrm{l}$ of icecold lysis buffer $(62.5 \mathrm{mM}$ TRIS- $\mathrm{HCl} \mathrm{pH} 6.8,1 \%$ Triton $\mathrm{X}-100,1 \%$ deoxycholate, $50 \mathrm{mM} \mathrm{NaF}, 1 \mathrm{mM} \mathrm{Na} \mathrm{VO}_{3}$, cOmplete $^{\mathrm{TM}}$ Protease Inhibitor Cocktail (CO-RO) $(1$ tablet $/ 50 \mathrm{ml}$ of solution), Roche, Mannheim, Germany) and stored at $-80^{\circ} \mathrm{C}$ until homogenization for western blots (WB).

The subcutaneous adipose tissue (SCAT) and intraperitoneal adipose tissue (IPAT) were removed and weighed.

The liver was weighed and immersed in 4\% PFA, and after $24 \mathrm{~h}$, it was stored at $4{ }^{\circ} \mathrm{C}$ in $70 \%$ ethanol, embedded into paraffin blocks, and stored until cutting for histology.

\section{Biochemical parameters}

The concentration of fasting plasma insulin was measured by RIA assay (Millipore, St. Charles, MI, USA). The concentration of free fatty acids (FFA) in plasma samples was determined using a colorimetric assay (Roche, Mannheim, Germany). The concentration of cholesterol in the plasma samples was determined using a colorimetric assay kit (Erba Lachema, Brno, Czech Republic). Plasma triglyceride (TG) levels were measured using a quantitative enzymatic reaction kit (Sigma, St. Louis, MO, USA). The leptin concentration in plasma samples was determined by ELISA (Millipore, St. Charles, MI, USA). All measurements were performed according to the manufacturer's instructions.

\section{Insulin resistance quantification}

The homeostatic model assessment for insulin resistance (HOMA-IR) and quantitative insulin sensitivity check index (QUICKI) were calculated from the measured fasting plasma glucose and fasting plasma insulin concentrations as described previously [23, 24]. Briefly, HOMA-IR and QUICKI were calculated using the following equations: HOMA-IR $=\{$ (fasting plasma glucose $)[\mathrm{mmol} / \mathrm{l}] \times$ (fasting plasma insulin) $[\mathrm{pmol} / 1]\} / 22.5$; QUICKI $=1 /$ $\left[\left(\log \left(\mathrm{I}_{0}\right)+\log \left(\mathrm{G}_{0}\right)\right]\right.$, where $\mathrm{I}_{0}$ is the fasting plasma insulin level $[\mu \mathrm{U} / \mathrm{ml}]$, and $\mathrm{G}_{0}$ is the fasting blood glucose level $[\mathrm{mg} / \mathrm{dl}]$.

\section{Liver histology}

The liver slices were stained with hematoxylin and eosin for histological analysis as described previously [4].

\section{Brain immunohistochemistry}

PFA-fixed right brain hemispheres were cut according to Holubová et al. [22]. The sections were kept in cryoprotective solution $(30 \%(\mathrm{v} / \mathrm{v})$ ethylene glycol, $25 \%(\mathrm{v} / \mathrm{v})$ glycerol, $45 \%$ (v/v) $\mathrm{pH} 7.41 \times \mathrm{PBS})$ at $-20^{\circ} \mathrm{C}$ until immunohistochemistry staining.

3,3'-Diaminobenzidine (DAB) staining with peroxidase rabbit IgG was performed as described previously [22]. Fluorescence staining was performed the same with the following modifications: the primary antibody (Table 2) was diluted in $3 \%$ normal goat serum (NGS). In the final step of staining, the sections were washed 2 times with TBS-T and 1 time with $1 \times$ TBS, mounted on silanized slides, and coverslipped using Vectashield ${ }^{\circ}$ Antifade Mounting Medium with 4',6'-diamidin-2-phenylindole (DAPI) (Vector Laboratories, Inc., Burlingame, CA, USA). The samples were stored in the dark at $4{ }^{\circ} \mathrm{C}$ until imaging. The list of secondary antibodies is in Table 3. Fluorescence staining was used for the colocalization of activated astrocytes (glial fibrillary acidic protein, GFAP), phosphorylated tau at the T231 residue (pTau (T231)) (AT180), and cell nuclei (DAPI).

Stained sections were imaged by an IX83 P1ZF Olympus Microscope (Olympus Corporation, Tokyo, Japan) equipped with a DP74 camera with bright field or fluorescence CoolLed pE-Universal Collimator sources using OLYMPUS CellSens Dimension software. Six to seven approximately same size middle-part sections per brain ( $\mathrm{n}=4$ randomly chosen from each group) were analyzed for DAB staining. The area of interest was selected manually according to the mouse brain atlas (The mouse brain in stereotaxic coordinates, Paxinos and Franklin, 1997). Using ImageJ software (NIH, Bethesda, MD, USA), the areas of interest of stained sections were analyzed as a percentage of a dark background from the area of interest (the hippocampus, cortex, and amygdala for ionized calcium-binding adapter molecule 1 (Iba1), and the hippocampus and cortex for GFAP) divided by the average percentage of a dark background from the area of interest of wt mice on the St diet. The threshold was kept constant for all samples in the particular staining experiment. All areas of interests were approximately the same size across the chosen brain sections.

The fluorescence-stained sections were not quantified. Illustrative pictures were chosen for the presentation. 
Table 2 Primary antibodies used at immunohistochemistry

\begin{tabular}{llll}
\hline Primary antibody & Dilution & DAB staining & Manufacturer \\
\hline GFAP rabbit pAb & $1: 200$ & $1 \mathrm{~min}, 10 \mathrm{sec}$ & Thermofisher, Rockford, IL, USA \\
Iba1 rabbit pAb & $1: 2000$ & 3 mins & FUJIFILM Wako Pure Chemical Corp., Richmond, VA, USA \\
AT180 mouse mAb & $1: 400$ & - & Thermofisher, Rockford, IL, USA \\
\hline
\end{tabular}

$D A B$ 3,3'-diaminobenzidine, GFAP glial fibrillary acidic protein, Iba1 ionized calcium-binding adapter molecule 1

\section{Western blotting}

The hippocampi ( $\mathrm{n}=6$ mice per group) were homogenized in $200 \mu \mathrm{l}$ of ice-cold lysis buffer using a Bullet Blender homogenizer (Next Advance, Inc. Averill Park, NY, USA). The homogenates were sonicated for $1 \mathrm{~min}$ and centrifuged for $8 \mathrm{~min}$ at $3080 \times g$ at $4{ }^{\circ} \mathrm{C}$. The supernatant protein concentrations were determined using a Pierce BCA protein assay kit (Thermo Fisher Scientific, Waltham, MA, USA). The supernatant samples (7 $\mu \mathrm{g}$ of protein $/ 7 \mu$ l of sample/line) were prepared and resolved according to Holubová et al. [22]. The list of primary antibodies used is shown in Table 4. Transferred protein bands on nitrocellulose membrane (Bio-Rad, Hercules, CA, USA) were visualized by ChemiDoc ${ }^{\mathrm{TM}}$ MP System (Bio-Rad, Hercules, CA, USA) and quantified using Image Lab Software (Bio-Rad, Hercules, CA, USA) as described previously [22].

\section{Statistical analyses}

The data are presented as the mean \pm SEM and were analyzed with GraphPad Prism 8.0 Software (San Diego, CA, USA) using the nonparametric unpaired Mann-Whitney $t$ test for particular pair comparison (wt vs THY-Tau22, St vs HF diet) within each age and sex group and nonparametric unpaired mixed-effects analysis of two-way ANOVA and Bonferroni's post hoc test for age comparison as stated in the figure and table legends. The area under the curve (AUC $C_{\Delta \text { Glucose }}$ ) was statistically analyzed using nonparametric unpaired Kruskal-Wallis one-way ANOVA and Bonferroni's post hoc test within each age and sex group. $\mathrm{p}<0.05$ was considered statistically significant.

\section{Results}

The combination of age and a HF diet significantly worsened the mobility of mice

Age significantly decreased mobility, indicated by a decreased average velocity and running distance, in all older groups compared to the younger groups (Fig. 1A).
The 11-month-old mice also showed a higher anxietylike tendency, which was expressed by a shorter mean wall distance (Supplementary Fig. 1). Furthermore, the HF diet slightly or significantly decreased the mobility of all mouse groups, except for 7-month-old THY-Tau22 females and 11-month-old wt females. Out of all groups, the 11-month-old THY-Tau22 females fed a HF diet showed the lowest mobility. Interestingly, the HF diet caused a significant increase of anxiety-like behavior of THY-Tau22 females when compared to wt controls expressed by the lowest wall distance (Supplementary Fig. 1).

\section{The combination of age and THY-Tau22 genotype significantly worsened short-term spatial memory in males}

Female groups showed no differences in the time spent in the NZ of the Y-maze (Fig. 1B). In contrast, 7- and 11-month-old THY-Tau22 males fed the St diet showed a poor willingness to spend time in the NZ when compared to wt controls (Fig. 1B). Furthermore, 11-monthold THY-Tau22 males on HF diet showed an even stronger decrease when compared to the same diet wt controls (Fig. 1B); nevertheless, the number of NZ visits for 11-month-old THY-Tau22 males did not show a significant decrease when compared to the same diet wt controls (Fig. 1B). The number of NZ visits significantly decreased in 7- and 11-month-old males on St diet when compared to wt controls (Fig. 1B).

\section{HF diet significantly increased the body weight and the fat weight of mice}

The HF diet significantly increased the body weight of all mice (Fig. 2). The body weights of THY-Tau22 males and females fed the St diet were lower than those of their respective wt controls. The same trend was observed in males but not females fed the HF diet (Fig. 2).

Table 3 Secondary antibodies used at immunohistochemistry

\begin{tabular}{llll}
\hline Staining & Secondary antibody & Dilution & Manufacturer \\
\hline DAB & Peroxidase rabbit lgG & $1: 400$ & Vector Lab., Burlingame, CA, USA \\
Fluorescence & AlexaFluor 488 mouse pAb & $1: 1000$ & Thermofisher, Rockford, IL, USA \\
& AlexaFluor 568 rabbit pAb & $1: 1000$ & Thermofisher, Rockford, IL, USA \\
\hline
\end{tabular}


Table 4 Primary antibodies used at western blots of hippocampi

\begin{tabular}{|c|c|c|c|}
\hline Primary antibody & Dilution & Diluent & Manufacturer \\
\hline$\beta$-actin mouse mAb & $1: 10,000$ & $5 \%$ milk & Sigma-Aldrich, St. Louis, MO, USA \\
\hline Akt rabbit $\mathrm{mAb}$ & $1: 1000$ & $5 \%$ BSA & Cell Signaling Technology, Danvers, MA, USA \\
\hline pAkt (T308) rabbit mAb & $1: 1000$ & $5 \%$ BSA & Cell Signaling Technology, Danvers, MA, USA \\
\hline pAkt (S473) rabbit mAb & $1: 1000$ & $5 \%$ BSA & Cell Signaling Technology, Danvers, MA, USA \\
\hline CD11b rabbit mAb & $1: 1000$ & $5 \%$ milk & Abcam, Cambridge, UK \\
\hline p44/42 ERK1/2 mouse mAb & $1: 2000$ & $5 \%$ milk & Cell Signaling Technology, Danvers, MA, USA \\
\hline p44/42 pERK1/2 (T202/Y204) mouse mAb & $1: 2000$ & $5 \%$ milk & Cell Signaling Technology, Danvers, MA, USA \\
\hline GFAP mouse mAb & $1: 1000$ & $5 \%$ milk & Cell Signaling Technology, Danvers, MA, USA \\
\hline GSK-3 $\beta$ rabbit mAb & $1: 1000$ & $5 \%$ BSA & Cell Signaling Technology, Danvers, MA, USA \\
\hline pGSK-3 $\beta$ (S9) rabbit mAb & $1: 1000$ & $5 \%$ BSA & Cell Signaling Technology, Danvers, MA, USA \\
\hline PDK-1 rabbit pAb & $1: 1000$ & $5 \%$ BSA & Cell Signaling Technology, Danvers, MA, USA \\
\hline pPDK-1 (S241) rabbit mAb & $1: 1000$ & $5 \%$ BSA & Cell Signaling Technology, Danvers, MA, USA \\
\hline PI3Kp85 rabbit mAb & $1: 1000$ & $5 \%$ BSA & Cell Signaling Technology, Danvers, MA, USA \\
\hline PP2A C rabbit mAb & $1: 1000$ & $5 \%$ BSA & Cell Signaling Technology, Danvers, MA, USA \\
\hline methyl-PP2A C (L309) mouse mAb & $1: 1000$ & $5 \%$ milk/ $1 \%$ BSA & Millipore, Temecula, CA, USA \\
\hline pPP2A (Y307) rabbit mAb & $1: 5000$ & $5 \%$ milk & Abcam, Cambridge, UK \\
\hline PSD95 rabbit pAb & $1: 1000$ & $5 \%$ BSA & Cell Signaling Technology, Danvers, MA, USA \\
\hline Synaptophysin rabbit pAb & 1:5000 & $5 \%$ milk & Santa Cruz Biotechnology, Inc., Dallas, TX, USA \\
\hline Tau 5 mouse mAb & 1:5000 & $5 \%$ milk & Invitrogen Corp., Frederick, MD, USA \\
\hline pTau (pThr212) rabbit pAb & 1:5000 & $5 \%$ BSA & Thermofisher, Rockford, IL, USA \\
\hline pTau (pSer214) rabbit pAb & $1: 1000$ & $5 \%$ BSA & Thermofisher, Rockford, IL, USA \\
\hline pTau (pThr231) rabbit pAb & $1: 10,000$ & $5 \%$ BSA & Thermofisher, Rockford, IL, USA \\
\hline pTau (pSer396) rabbit pAb & $1: 10,000$ & $5 \%$ BSA & Thermofisher, Rockford, IL, USA \\
\hline pTau (pSer404) rabbit pAb & $1: 1000$ & $5 \%$ BSA & Thermofisher, Rockford, IL, USA \\
\hline pTau (pSer422) rabbit pAb & $1: 1000$ & $5 \%$ BSA & Thermofisher, Rockford, IL, USA \\
\hline
\end{tabular}

$B S A$ bovine serum albumin, $m A b$ monoclonal antibody, $p A b$ polyclonal antibody All diluents are diluted in TBS/Tween-20; non-fat dry milk was used

Accordingly, the weights of SCAT and IPAT were significantly increased by the HF diet in 7- and 11-month-old mice of both sexes (Fig. 3). Furthermore, 7- and 11-monthold THY-Tau22 males on the St diet showed lower weights of SCAT and IPAT than their respective wt controls (Fig. 3). The weight of SCAT correlated with the weight of IPAT in 7- but not 11-month-old animals of both sexes (Fig. 3).

Furthermore, the HF diet-induced obesity led to a significant increase of $\mathrm{C}$-reactive protein (CRP), the inflammation marker, in the fasting plasma of 11-month-old animals, when compared to the St diet-fed animals (Fig. 4).

\section{HF diet caused liver steatosis}

HF diet feeding generally caused a significant increase in liver weight and typical liver steatosis in all 11-monthold mice (Fig. 5). Representative figures of liver slices from 11-month-old transgenic males and females on both diets are shown in Fig. 5B and $\mathrm{C}$, respectively. In contrast, St diet-fed mice did not show any evidence of liver steatosis, even at a late age (Fig. 5).
HF diet significantly increased plasma leptin and lipids The HF diet increased the plasma level of leptin very substantially in all 7- and 11-month-old mice, in line with body weight and SCAT and IPAT weight (Tables 5, 6, and 7; Figs. 2 and 3). A similar trend was observed for plasma cholesterol, except for 7-month-old males (Tables 5, 6, and 7).

Unexpectedly, the THY-Tau22 genotype caused a significant increase in FFA in 3-month-old females. The HF diet enhanced plasma FFA in all 11-month-old but not in 7-month-old mice, where no difference in FFA among groups was observed. TG levels were increased ambiguously in some groups (Tables 5, 6, and 7).

\section{No significant differences among groups were detected in tolerance to glucose}

Fasting glucose levels were normal at 3 months of age in all groups. At 7 months, the fasting glucose level increased nondramatically above the normoglycemic value (the value for lean wt mice), but at 11 months of age, it did not 


\section{a Open field}
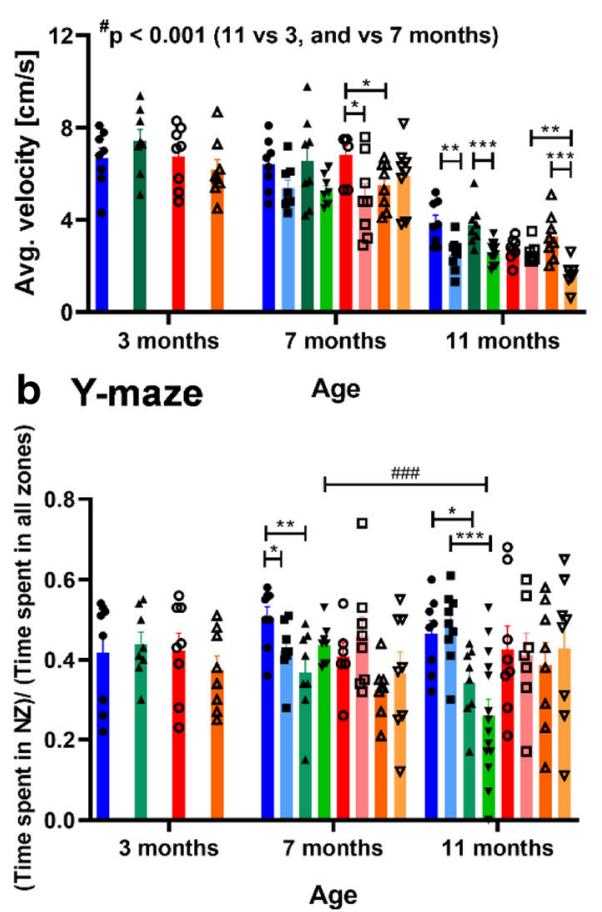

Males, wt, St

Males, wt, HF

Males, Tau22, St

Males, Tau22, HF

Females, wt, St

Females, wt, HF

Females, Tau22, St

Females, Tau22, HF

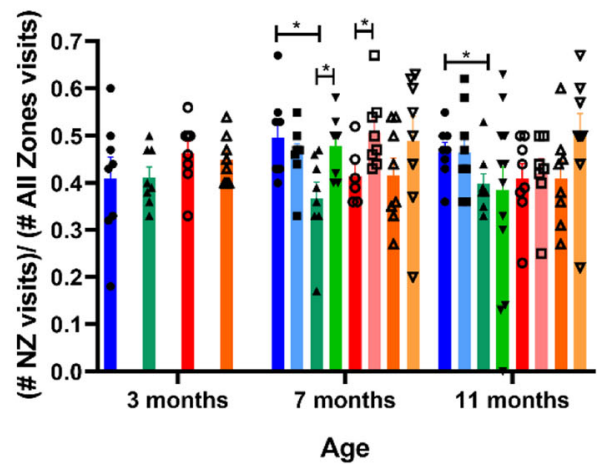

Fig. 1 Behavioral tests. a Open field $(n=7-14)$. b Y-maze $(n=6-14)$. Data are presented as mean \pm SEM and were statistically analyzed by Mann-Whitney t test $\left(^{*}\right)$ within each age and sex group $\left({ }^{*} p<0.05 ;{ }^{* *} p<0.01\right)$. The age comparison was performed by mixed-effects analysis and Bonferroni's post hoc test $(\#)$. A value significance between 11 and 3 , and 11 and 7 months in a particular group at an average velocity of open field test was \#\#\#<0.001 in all cases (not shown for clarity). NZ, new zone

rise again. HF-diet feeding increased fasting glucose only in 7-month-old wt males and females (Tables 5, 6, and 7).

The OGTT AUC values did not show any significant differences among the tested groups, both 7- and 11month-old mice (Fig. 6).

\section{HF diet-fed mice showed increased peripheral insulin resistance}

The HF diet significantly increased the concentration of fasting plasma insulin in all groups (Table 8). Even though fasting glucose was increased only slightly by the
HF diet, all mice fed the HF diet showed a significantly higher HOMA-IR index than their respective St diet-fed controls (Table 8), which indicates increased peripheral IR. HOMA-IR indexes also increased in 11-month-old wt males on the St diet and THY-Tau22 females on the HF diet compared to the respective 7-month-old controls (Table 8).

The HF diet also caused a significant decrease in the QUICKI index in all mice. There was a significant increase in the QUICKI index between 7 and 11 months in wt females on the St diet (Table 8).
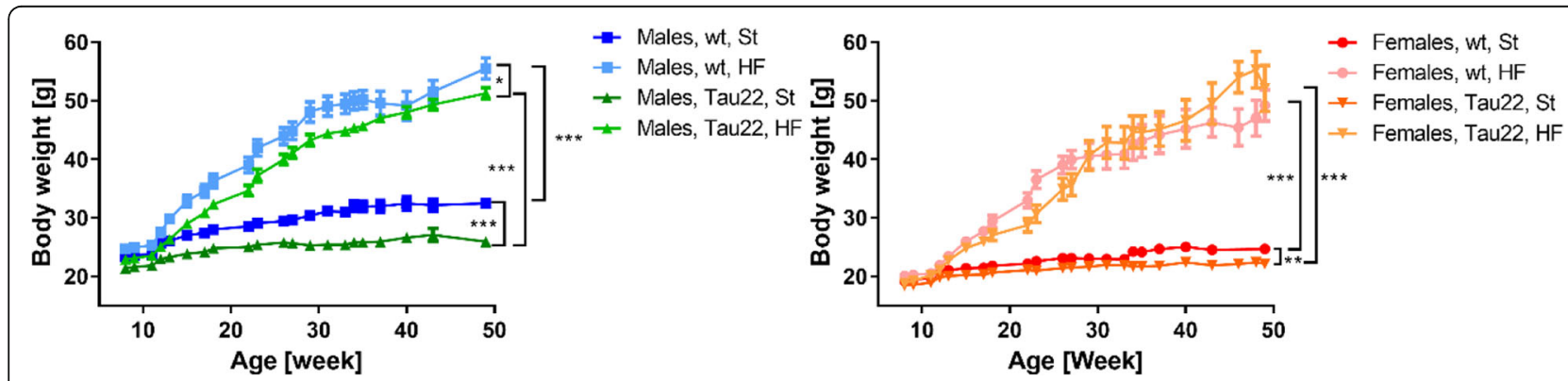

Fig. 2 Body weight of THY-Tau22 and wt mice. Males: $n=8-23$, females: $7-21$. Data are presented as mean \pm SEM. The comparison of final weights (males: $\mathrm{n}=8-14$, females: $7-8$ ) was statistically analyzed by Mann-Whitney t test $\left({ }^{*} p<0.05 ;{ }^{* *} p<0.01 ;{ }^{* * *} p<0.001\right.$ ) 


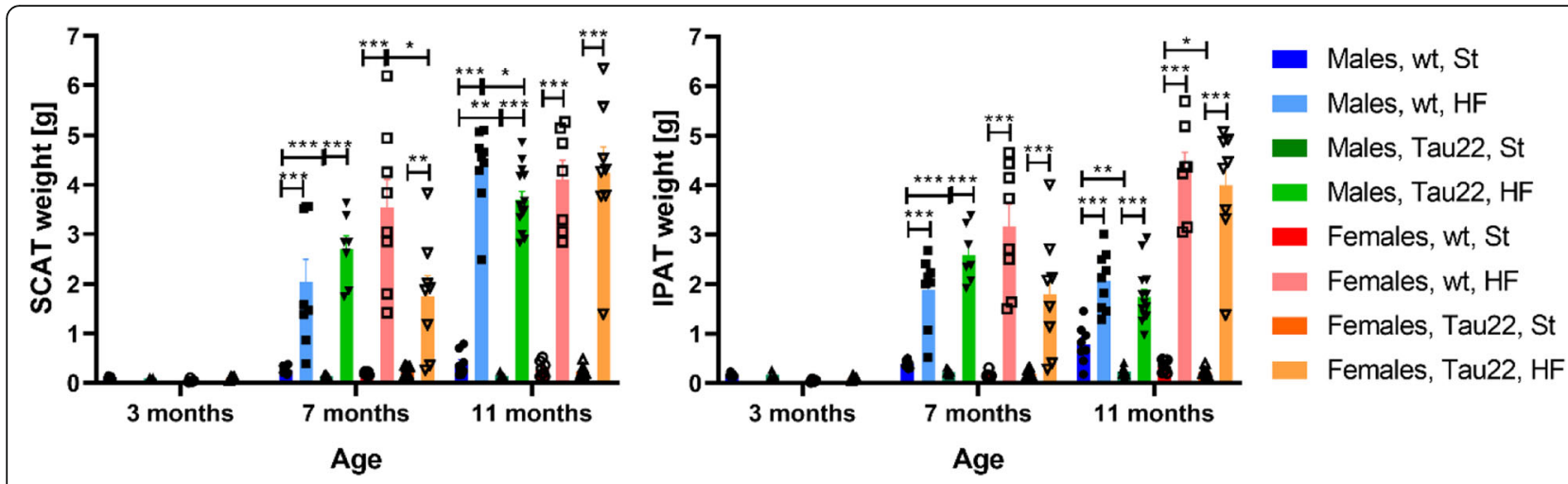

Fig. 3 Adipose tissues weight of THY-Tau22 and wt mice. Subcutaneous (SCAT) and intraperitoneal (IPAT) adipose tissue weight of 3- $(n=4-5)$, 7- $(n=7-8)$, and 11-month-old $(n=7-14)$ mice. Data are presented as mean \pm SEM and were statistically analyzed by Mann-Whitney $t$ test within each age and sex group $\left({ }^{*} p<0.05 ;{ }^{* *} p<0.01\right.$; ${ }^{* *} p<0.001$ )

THY-Tau22 genotype and HF diet significantly increased neuroinflammation

Neuroinflammation is typically characterized by a higher number of clusters of microglia (marked by Iba1) and activated astrocytes (marked by GFAP).

Iba1 differed among groups of the same age in males and females. Among the 7-month-old males, only the THY-Tau22 males on the St diet showed increased Iba1 levels in the hippocampus and amygdala compared to wt controls. In females of the same age, Iba1 was increased significantly in the amygdala in THY-Tau22 females on the St diet compared to wt females. Furthermore, the HF diet increased Iba1 in the hippocampus and cortex of 7-month-old wt females. However, in 11-month-old females, nearly no difference in Iba1 was registered among groups in all three areas. On the other hand, 11- month-old THY-Tau22 males fed the HF diet showed massively increased Iba1 in all three areas compared to THY-Tau22 males fed the St diet or wt HF diet. In addition, Iba1 was significantly increased in 11-monthold THY-Tau22 males fed the HF diet compared to those 7-month-old fed the HF diet (Fig. 7, Supplementary Fig. 2). WB performed in the hippocampus labeled with anti-CD11b antibody showed only one significant increase in 7-month-old transgenic females fed the HF diet when compared to wt females fed the same diet (Supplementary Fig. 2).

Seven-month-old males did not show any significant difference in hippocampal GFAP detected by IHC, as did females (Fig. 8F). In contrast, the WB of hippocampal GFAP showed increased GFAP in 7-month-old THYTau22 males on the St diet and 11-month-old THY-

\section{1 months, males}
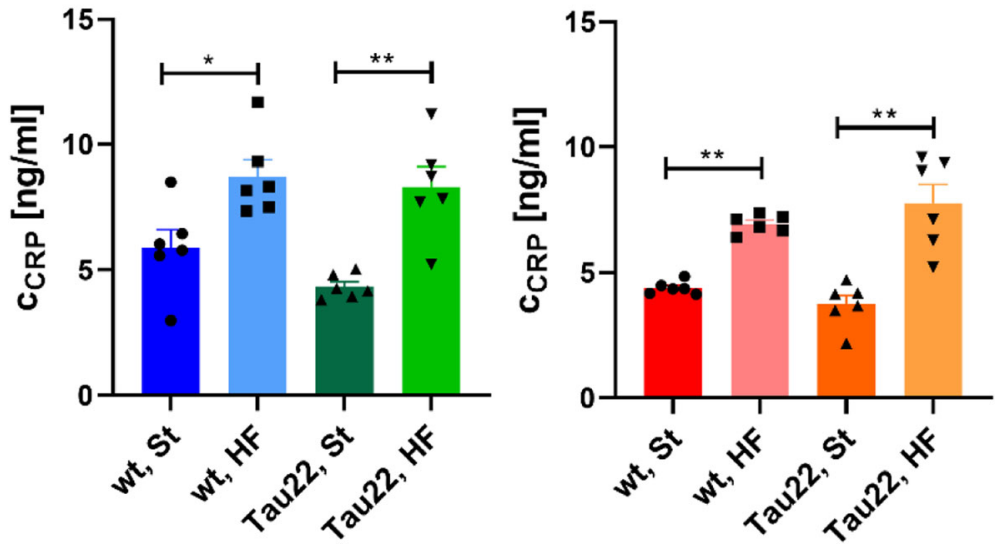

Fig. 4 Inflammation marker in the blood plasma. The concentration of C-reactive protein (CRP) in non-anesthetized fasting plasma of 11-monthold male and female mice $(n=6)$. Data are presented as mean \pm SEM and were statistically analyzed by Mann-Whitney $t$ test within each age and sex group $\left({ }^{*} p<0.05 ;{ }^{* *} p<0.01\right)$ 


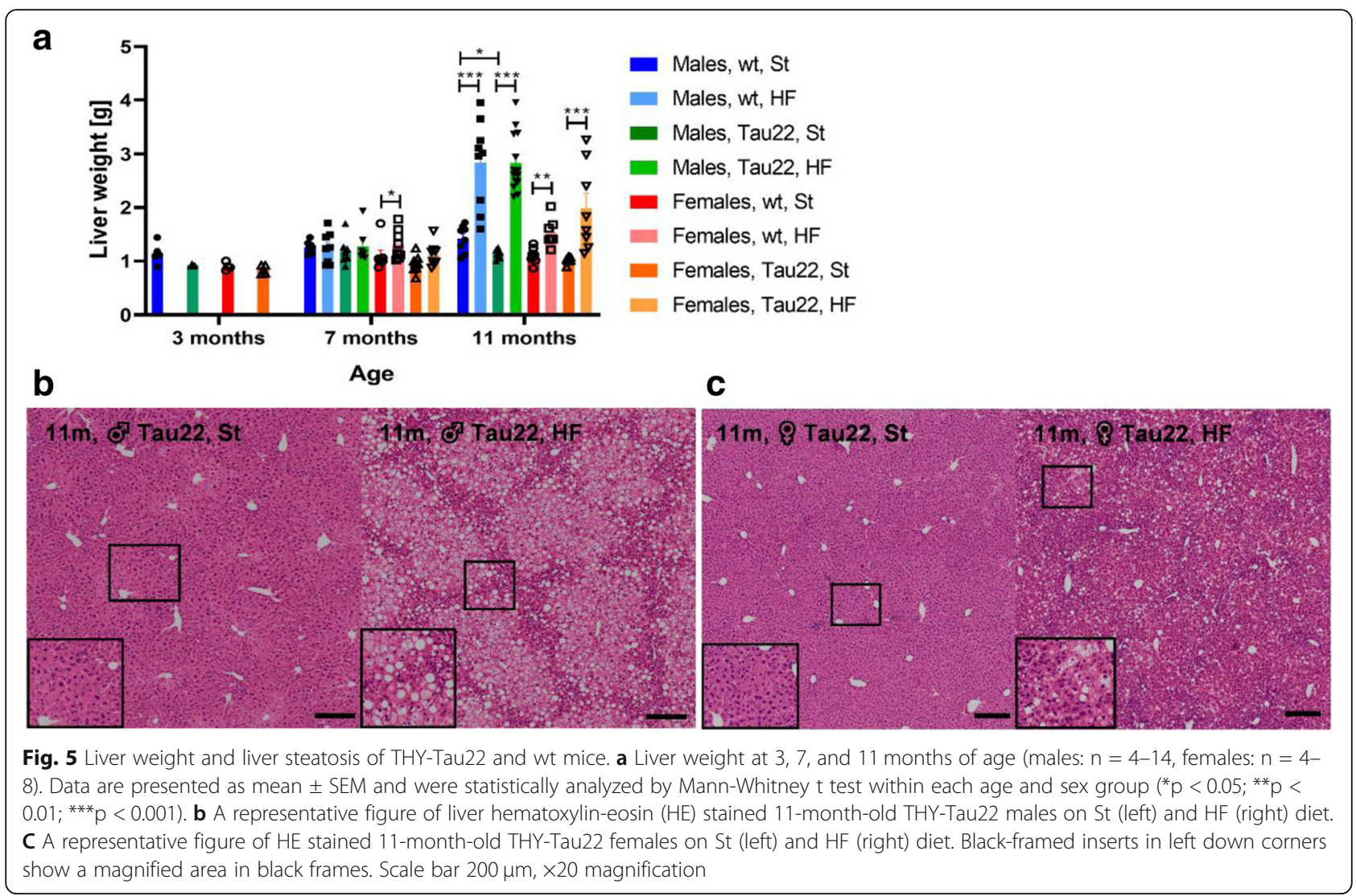

Table 5 Metabolic parameters of THY-Tau22 and wt mice. Three-month-old

\begin{tabular}{llll}
\hline Parameter & Sex & wt St & Tau22 St \\
\hline Fasting glucose $[\mathrm{mmol} / \mathrm{l}](\mathrm{n}=4-5)$ & Male & $6.32 \pm 0.30$ & $5.93 \pm 0.36$ \\
Cholesterol $[\mathrm{mmol} / \mathrm{l}](\mathrm{n}=4-5)$ & Male & $1.24 \pm 0.07$ & $1.20 \pm 0.06$ \\
FFA $[\mathrm{ng} / \mathrm{ml}](\mathrm{n}=4-5)$ & Male & $0.24 \pm 0.08$ & $0.34 \pm 0.06$ \\
TG $[\mathrm{mmol} / \mathrm{l}](\mathrm{n}=4-5)$ & Male & $0.22 \pm 0.05$ & $0.30 \pm 0.04$ \\
Leptin $[\mathrm{ng} / \mathrm{ml}](\mathrm{n}=4-5)$ & Male & $0.59 \pm 0.06$ & $0.51 \pm 0.03$ \\
Fasting glucose $[\mathrm{mmol} / \mathrm{l}](\mathrm{n}=4-5)$ & Female & $5.30 \pm 0.43$ & $4.00 \pm 0.28^{+}$ \\
Cholesterol $[\mathrm{mmol} / \mathrm{l}](\mathrm{n}=4-5)$ & Female & $0.97 \pm 0.09$ & $1.01 \pm 0.08$ \\
FFA $[\mathrm{ng} / \mathrm{ml}](\mathrm{n}=4-5)$ & Female & $0.10 \pm 0.02$ & $0.61 \pm 0.09^{+}$ \\
TG $[\mathrm{mmol} / \mathrm{l}](\mathrm{n}=4-5)$ & Female & $0.25 \pm 0.04$ & $0.25 \pm 0.03$ \\
Leptin $[\mathrm{ng} / \mathrm{ml}](\mathrm{n}=4-5)$ & Female & $0.52 \pm 0.08$ & $0.87 \pm 0.18$ \\
\hline
\end{tabular}

Fasting glucose, cholesterol, triglycerides (TG), free fatty acids (FFA), and leptin were measured in 3-, 7-, and 11-month-old males and females of both diets. The data are presented as mean \pm SEM and were statistically analyzed by Mann-Whitney t test within each age and sex group ( ${ }^{*} \mathrm{HF}$ vs St within the wt or Tau22 group, "Tau22 vs wt on the same diet). The age comparison was performed using mixed-effects analysis and Bonferroni's post hoc test ${ }^{\#} 7$ or 11 vs 3 months, \$11 vs 7 months). Statistical significance for all measurements: ${ }^{*} \mathrm{p}<0.05 ;{ }^{* *} \mathrm{p}<0.01 ;{ }^{* * *} \mathrm{p}<0.001$
Tau22 on both diets compared to wt males, but this effect was not observed in females (Fig. 8G and H). On the other hand, IHC results of 11-month-old THYTau22 males on both diets showed increased GFAP in the cortex compared to the wt males (Fig. 8F).

Finally, fluorescent staining was used for colocalization of activated astrocytes, pTau (T231), and neuron nuclei. The representative figures illustrate 7-month-old THYTau22 males and their wt controls, both on the St diet (Fig. 9A), and 11-month-old THY-Tau22 males and their wt controls, both on the HF diet (Fig. 9B). As shown in Fig. 9, pTau (T231) was abundantly present in the brains of THY-Tau22 males of both ages. GFAP was observed in close vicinity to pTau (T231), mostly in the amygdala and hippocampus. The abundance of pTau (T231) was also observed in the cortex of THY-Tau22 males (Fig. 9).

The combination of the THY-Tau22 genotype and HF diet significantly worsened hippocampal synaptic plasticity in 11-month-old female mice

Synaptophysin and postsynaptic density protein 95 (PSD95) are markers of synaptic and postsynaptic plasticity, respectively. A significant decrease in the relative intensity of PSD95 and synaptophysin was shown in the hippocampi of 11-month-old transgenic females on both 
Table 6 Metabolic parameters of THY-Tau22 and wt mice. Seven-month-old

\begin{tabular}{|c|c|c|c|c|c|}
\hline Parameter & Sex & wt St & wt HF & Tau22 St & Tau22 HF \\
\hline Fasting glucose [mmol/l] $(\mathrm{n}=7-8)$ & Male & $8.98 \pm 0.43^{\#}$ & $10.80 \pm 0.80^{*}$ & $9.39 \pm 1.03^{\#}$ & $10.73 \pm 0.72$ \\
\hline Cholesterol $[\mathrm{mmol} / \mathrm{l}](\mathrm{n}=7-8)$ & Male & $1.06 \pm 0.16$ & $1.64 \pm 0.26$ & $0.88 \pm 0.05$ & $1.87 \pm 0.13^{* * *}$ \\
\hline FFA [ng/ml] $(\mathrm{n}=7-8)$ & Male & $0.39 \pm 0.05$ & $0.48 \pm 0.07$ & $0.45 \pm 0.09$ & $0.77 \pm 0.07^{*+}+$ \\
\hline $\mathrm{TG}[\mathrm{mmol} / \mathrm{l}](\mathrm{n}=7-8)$ & Male & $0.48 \pm 0.11^{\#}$ & $0.63 \pm 0.12$ & $0.36 \pm 0.03$ & $0.78 \pm 0.08^{* * *}$ \\
\hline Leptin [ng/ml] ( $\mathrm{n}=7-8)$ & Male & $3.22 \pm 0.49$ & $70.76 \pm 19.41^{* *}$ & $1.68 \pm 0.60$ & $61.82 \pm 12.30^{* * *}$ \\
\hline Fasting glucose $[\mathrm{mmol} / \mathrm{l}](\mathrm{n}=7-8)$ & Female & $7.47 \pm 0.84$ & $9.61 \pm 0.38^{*}$ & $8.10 \pm 0.97^{\# \# \#}$ & $10.19 \pm 0.66$ \\
\hline Cholesterol $[\mathrm{mmol} / \mathrm{l}](\mathrm{n}=7-8)$ & Female & $0.57 \pm 0.03^{\# \#}$ & $1.23 \pm 0.08^{* * *}$ & $0.62 \pm 0.05^{\# \# \#}$ & $1.33 \pm 0.13^{* * *}$ \\
\hline FFA [ng/ml] $(n=7-8)$ & Female & $0.40 \pm 0.08^{\#}$ & $0.46 \pm 0.03$ & $0.52 \pm 0.08$ & $0.56 \pm 0.04$ \\
\hline $\mathrm{TG}[\mathrm{mmol} / \mathrm{l}](\mathrm{n}=7-8)$ & Female & $0.31 \pm 0.03$ & $0.46 \pm 0.03^{* *}$ & $0.58 \pm 0.28$ & $0.40 \pm 0.02$ \\
\hline Leptin [ng/ml] ( $\mathrm{n}=7-8)$ & Female & $3.19 \pm 0.80^{\#}$ & $92.22 \pm 14.35^{* * *}$ & $2.95 \pm 0.27$ & $42.06 \pm 12.20^{* * *}$ \\
\hline
\end{tabular}

Fasting glucose, cholesterol, triglycerides (TG), free fatty acids (FFA), and leptin were measured in 3-, 7-, and 11-month-old males and females of both diets. The data are presented as mean \pm SEM and were statistically analyzed by Mann-Whitney t test within each age and sex group (*HF vs St within the wt or Tau22 group, 'Tau22 vs wt on the same diet). The age comparison was performed using mixed-effects analysis and Bonferroni's post hoc test ( ${ }^{\sharp} 7$ or 11 vs 3 months, ${ }^{\$} 11$ vs 7 months). Statistical significance for all measurements: ${ }^{*} p<0.05 ;{ }^{* *} p<0.01 ;{ }^{* * *} p<0.001$

diets compared to their respective wt controls (Fig. 10). Furthermore, the HF diet itself significantly decreased synaptophysin in both THY-Tau22 and wt females. Interestingly, males of the same age did not show any significant difference in synaptic plasticity in the hippocampus among the groups (Fig. 10).

The THY-Tau22 genotype and/or HF diet can differentially affect hippocampal insulin signaling in male and female mice

Hippocampal Insulin Receptor $\beta$ (IR $\beta$ ) increased significantly in 11-month-old THY-Tau22 males fed both diets when compared to the corresponding controls. Interestingly, the HF diet-fed wt females showed lower hippocampal expression of IR $\beta$, although this increased and reached a similar level to the rest of the female groups in THY-Tau22 HF diet-fed females (Fig. 11). Although the HF diet significantly increased the p 85 regulatory subunit of phosphoinositide 3-kinase (PI3Kp85) in 11-monthold THY-Tau22 males and females, phosphoinositidedependent kinase-1 phosphorylation at the S241 residue (pPDK-1 (S241)) was decreased in THY-Tau22 males fed the HF diet. THY-Tau22 of both sexes on the St diet had higher pPDK-1 than the respective wt controls (Fig. 11, Supplementary Fig. 3).

The phosphorylation of protein kinase B (Akt) at the T308 residue is mediated by PDK-1. Consistent with the hippocampal pPDK-1 results, increased phosphorylated pAkt (T308) was observed in 11-month-old THY-Tau22 males, but the HF diet decreased pAkt (T308) in THYTau22 males (Fig. 11, Supplementary Fig. 3). The THYTau22 genotype led to a significant increase in

Table 7 Metabolic parameters of THY-Tau22 and wt mice. Eleven-month-old

\begin{tabular}{|c|c|c|c|c|c|}
\hline Parameter & Sex & wt St & wt HF & Tau22 St & Tau22 HF \\
\hline Fasting glucose $[\mathrm{mmol} / \mathrm{l}](\mathrm{n}=8-14)$ & Male & $7.74 \pm 0.69$ & $9.06 \pm 0.63$ & $7.11 \pm 0.58$ & $8.74 \pm 0.26^{*}$ \\
\hline Cholesterol [mmol/l] $(\mathrm{n}=7-14)$ & Male & $0.98 \pm 0.07$ & $3.25 \pm 0.17^{* * *}, \$ \$ \$$ & $1.00 \pm 0.05$ & $3.14 \pm 0.18^{* * *} \$ \$ \$$ \\
\hline FFA [ng/ml] $(n=8-13)$ & Male & $0.50 \pm 0.13$ & $0.67 \pm 0.06$ & $0.48 \pm 0.09$ & $0.83 \pm 0.06^{* *}$ \\
\hline $\mathrm{TG}[\mathrm{mmol} / \mathrm{l}](\mathrm{n}=7-14)$ & Male & $0.35 \pm 0.03$ & $0.53 \pm 0.04^{*}$ & $0.27 \pm 0.04$ & $0.49 \pm 0.04^{* * * * \$ \$}$ \\
\hline Leptin [ng/ml] ( $n=5-14)$ & Male & $4.71 \pm 2.06^{\#}$ & $42.59 \pm 1.63^{* * *}$ & $0.58 \pm 0.28^{+}$ & $47.47 \pm 1.05^{* * *++}$ \\
\hline Fasting glucose $[\mathrm{mmol} / \mathrm{l}](\mathrm{n}=7-8)$ & Female & $7.70 \pm 0.42$ & $6.13 \pm 0.30^{*}, \$ \$ \$$ & $7.76 \pm 0.34^{\# \#}$ & $7.88 \pm 0.55^{+, \$}$ \\
\hline Cholesterol $[\mathrm{mmol} / \mathrm{l}](\mathrm{n}=7-8)$ & Female & $0.72 \pm 0.05$ & $1.92 \pm 0.13^{* * *, \$ \$ \$}$ & $0.76 \pm 0.09^{\#}$ & $2.04 \pm 0.16^{* * *, \$ \$ \$}$ \\
\hline $\mathrm{FFA}[\mathrm{ng} / \mathrm{ml}](\mathrm{n}=6-8)$ & Female & $0.41 \pm 0.04^{\#}$ & $0.68 \pm 0.07^{* *}$ \$ & $0.34 \pm 0.04^{\#, \$}$ & $0.57 \pm 0.04^{* *}$ \\
\hline $\mathrm{TG}[\mathrm{mmol} / \mathrm{l}](\mathrm{n}=7-8)$ & Female & $0.38 \pm 0.05$ & $0.56 \pm 0.05^{* *}$ & $0.39 \pm 0.04$ & $0.39 \pm 0.04^{+}$ \\
\hline Leptin [ng/ml] (n=7-8) & Female & $4.63 \pm 0.82^{\# \#}$ & $38.40 \pm 1.65^{* * *}, \$ \$ \$$ & $3.36 \pm 0.76^{\#}$ & $42.09 \pm 3.92^{* * *}$ \\
\hline
\end{tabular}

Fasting glucose, cholesterol, triglycerides (TG), free fatty acids (FFA), and leptin were measured in 3-, 7-, and 11-month-old males and females of both diets. The data are presented as mean \pm SEM and were statistically analyzed by Mann-Whitney $t$ test within each age and sex group ( ${ }^{*} \mathrm{HF}$ vs St within the wt or Tau22 group, 'Tau22 vs wt on the same diet). The age comparison was performed using mixed-effects analysis and Bonferroni's post hoc test $\left({ }^{\sharp} 7\right.$ or $11 \mathrm{vs} 3$ months, ${ }^{\$} 11$ vs 7 months). Statistical significance for all measurements: ${ }^{*} p<0.05 ;{ }^{* *} p<0.01 ;{ }^{* * *} p<0.001$ 


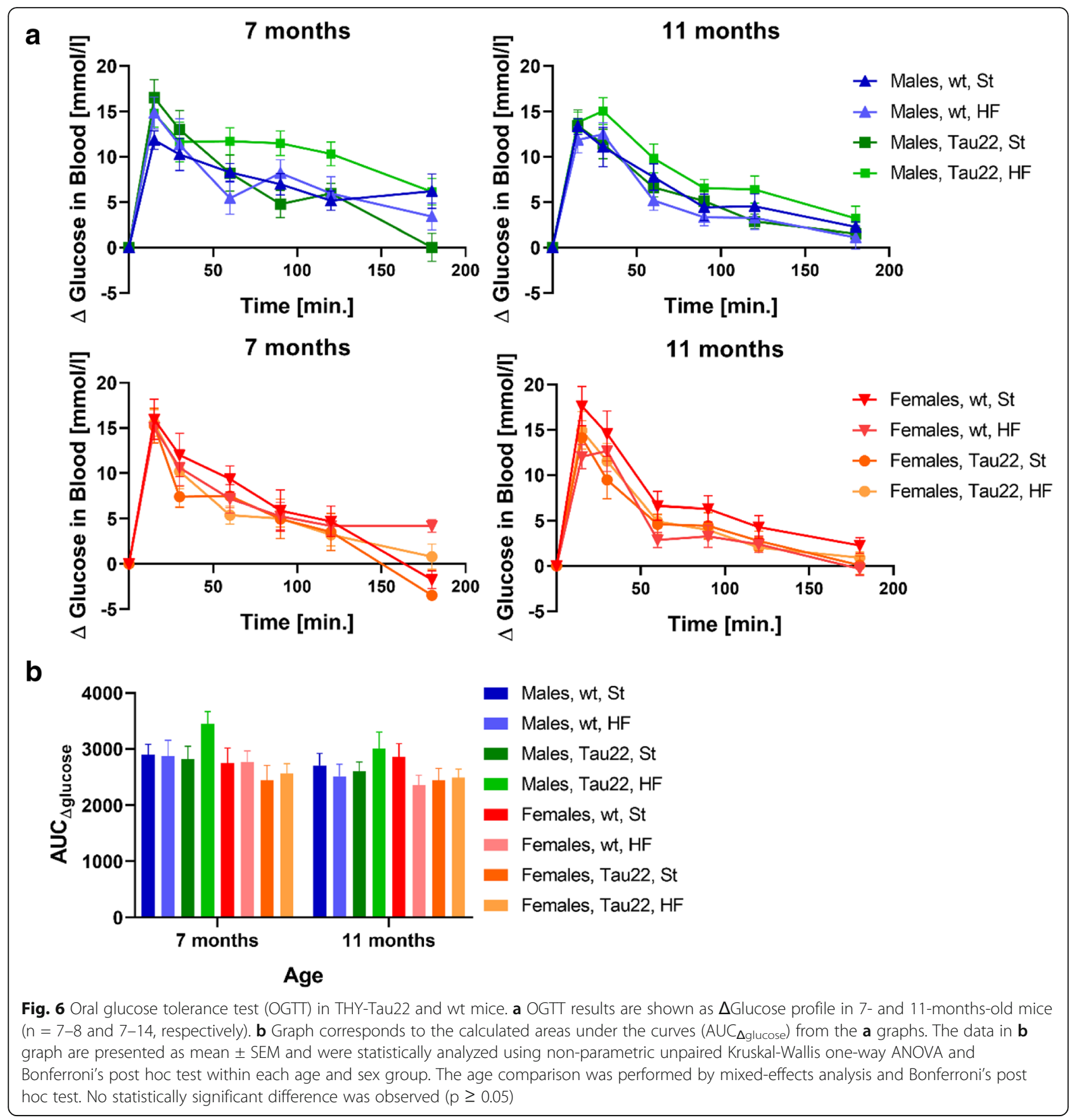

phosphorylated Akt at residue S473 (pAkt (S473)) in males and females fed the HF diet (Fig. 11, Supplementary Fig. 3). However, inhibitory pGSK-3 $\beta$ (S9) was increased, and thus, its kinase activity was attenuated in transgenic males fed the HF diet compared to the wt controls (Fig. 11, Supplementary Fig. 3).

Tau phosphorylation in THY-Tau22 mice is independent of ERK1/2 and PP2A activity

The HF diet significantly increased hippocampal extracellular signal-regulated kinase $1 / 2$ phosphorylation at
T202/Y204 residues (pERK1/2 (T202/Y204)) in THYTau22 females but not males (Fig. 12, Supplementary Fig. 4).

Hippocampal PP2A C was increased by HF diet feeding in THY-Tau22 males but not in females. The HF diet decreased the inactivated form of PP2A C, i. e. pPP2A C (Y307), in THY-Tau22 males. No further impact of the HF diet on the activity of PP2A was observed (Fig. 12).

Table 9 shows an overview of kinases and PP2A phosphatase used in this study, which have been found to 
Table 8 Insulin concentration and HOMA-IR and QUICKI indexes as insulin resistance quantification of THY-Tau22 and wt mice

\begin{tabular}{lllllll}
\hline & Sex & Age [months] & wt St & wt HF & Tau22 St & Tau22 HF \\
\hline Fasting insulin $[\mathrm{ng} / \mathrm{ml}]$ & Male $(n=7-8)$ & 7 & $0.18 \pm 0.02$ & $1.07 \pm 0.43^{* *}$ & $0.15 \pm 0.03$ & $1.17 \pm 0.32^{* * *}$ \\
& Male $(n=7-12)$ & 11 & $0.40 \pm 0.11^{\$}$ & $7.80 \pm 3.96^{* *} \$$ & $0.15 \pm 0.05$ & $2.43 \pm 0.85^{* * *}$ \\
& Female $(n=7-8)$ & 7 & $0.17 \pm 0.03$ & $0.57 \pm 0.14^{* *}$ & $0.12 \pm 0.03$ & $0.35 \pm 0.06^{* *}$ \\
& Female $(n=6-7)$ & 11 & $0.15 \pm 0.07$ & $1.87 \pm 0.82^{* *}$ & $0.22 \pm 0.06$ & $3.58 \pm 1.57^{* *}, \$ \$$ \\
HOMA-IR & Male $(n=7-8)$ & 7 & $12.45 \pm 1.65$ & $89.43 \pm 36.83^{* * *}$ & $10.81 \pm 2.83$ & $102.47 \pm 32.20^{* *}$ \\
& Male $(n=7-12)$ & 11 & $23.66 \pm 6.00$ & $475.67 \pm 210.86^{* *} \$$ & $8.53 \pm 2.85$ & $150.95 \pm 47.97^{* * *}$ \\
& Female $(n=7-8)$ & 7 & $10.22 \pm 2.37$ & $41.69 \pm 9.68^{* *}$ & $7.36 \pm 1.86$ & $26.52 \pm 4.33^{* *}$ \\
QUICKI & Female $(n=6-7)$ & 11 & $9.08 \pm 4.12$ & $83.87 \pm 32.06^{* *}$ & $14.16 \pm 4.31$ & $229.88 \pm 106.68^{* *} \$ \$$ \\
& Male $(n=7-8)$ & 7 & $0.35 \pm 0.01$ & $0.29 \pm 0.01^{* * *}$ & $0.38 \pm 0.02$ & $0.28 \pm 0.01^{* *}$ \\
& Male $(n=7-12)$ & 11 & $0.35 \pm 0.02$ & $0.25 \pm 0.01^{* *}$ & $0.39 \pm 0.01$ & $0.27 \pm 0.01^{* * *}$ \\
& Female $(n=7-8)$ & 7 & $0.37 \pm 0.01$ & $0.31 \pm 0.01^{* *}$ & $0.40 \pm 0.02$ & $0.32 \pm 0.01^{* *}$ \\
& Female $(n=6-7)$ & 11 & $0.55 \pm 0.12^{\$}$ & $0.29 \pm 0.01^{* *}$ & $0.36 \pm 0.02$ & $0.25 \pm 0.02^{* *}$ \\
\hline
\end{tabular}

Fasting insulin was measured in 7- and 11-month-old males and females of both diets. HOMA-IR and QUICKI indexes [23, 24] were calculated from the fasting insulin and fasting glucose concentrations (Table 5) for 7- and 11-month-old mouse groups. The data are presented as mean \pm SEM and were statistically analyzed by Mann-Whitney t test within each age and sex group (*HF vs St within the wt or Tau22 group). The age comparison was performed using mixedeffects analysis and Bonferroni's post hoc test ( ${ }^{\$} 11$ vs 7 months). Statistical significance for all measurements: ${ }^{*} p<0.05 ;{ }^{* *} p<0.01 ;{ }^{* * *} p<0.001$. HOMA-IR homeostatic model assessment for insulin resistance, QUICKI quantitative insulin sensitivity check index

directly de/phosphorylate tau protein at particular residues relevant for this study.

\section{The HF diet did not increase the relative intensity of phosphorylated tau proteins in transgenic mice}

The impact of the HF diet on the phosphorylated tau proteins, pTau (phosphorylated at T212, S214, T231, S396, S404, and S422) related to Tau5 (total tau) in 11month-old THY-Tau22 mice was determined. The results did not show any significant change in the relative pTau intensity for any epitope caused by the HF diet when compared to the St diet (Fig. 13, Supplementary Fig. 5).

\section{Discussion}

A number of studies have emphasized a link between obesity and central neuroinflammation leading to neurodegeneration in experimental rodent models and humans (reviewed in [2]). In this study, an agedependent effect of HF diet-induced obesity on potential brain neuroinflammation in THY-Tau22 mice, a model of tau pathology, was studied separately in males and females.

Open field data in this study suggested that just the impact of the higher age of mice was the strongest factor for lower mobility and higher anxiety, which was deepened in females by THY-Tau22 genotype and the HF diet. Regarding memory, THY-Tau22 males showed impaired long-term spatial memory in the Morris water maze at 9 months [9] and from 9 to 10 months of age [7]. The Y-maze test detected impaired short-term spatial memory in 12- to 13-month-old THY-Tau22 males compared to wt controls [8]. The current study showed significantly impaired short-term spatial memory in the Y-maze test in 7- and 11-month-old THYTau22 males but not females compared to the respective wt controls. The decrease in short-term memory was in line with the increase in neuroinflammation, as discussed below, affecting more males than females and pointed to neuroinflammation as a possible cause of the memory deficit.

The body weight of THY-Tau22 mice fed the St diet was significantly lower than that of wt mice on St diet as expected [16]. Nevertheless, our other results showed the same trend for HF diet-fed males, but not females, where the body weight of THY-Tau22 females increased more than wt females on the HF diet. However, HF dietinduced obesity increased the weight of SCAT and IPAT and plasma cholesterol in 7- and 11-month-old mice and liver weight and plasma FFA in 11-month-old mice, and all these data pointed to the development of IR. In humans, the development of IR in muscle and the liver was linked to FFA originating mainly from excessive subcutaneous fat [30]. HOMA-IR and QUICKI indexes confirmed significant peripheral IR in all mice with HF diet-induced obesity in our study. Similarly, in our previous studies in C57BL/6 mice strain, the background for THY-Tau22 mice, after feeding the HF diet for several months we repeatedly registered not only very significant obesity but also glucose intolerance and an enhanced HOMA-IR index, which was more distinct in males [18, 23, 31]. However, our current study did not show different tolerances to glucose (tested by OGTT) among the 7- or 11-month-old groups. Moreover, in this study, only males but not females 11-month-old developed massive liver steatosis in the fatty liver as another 


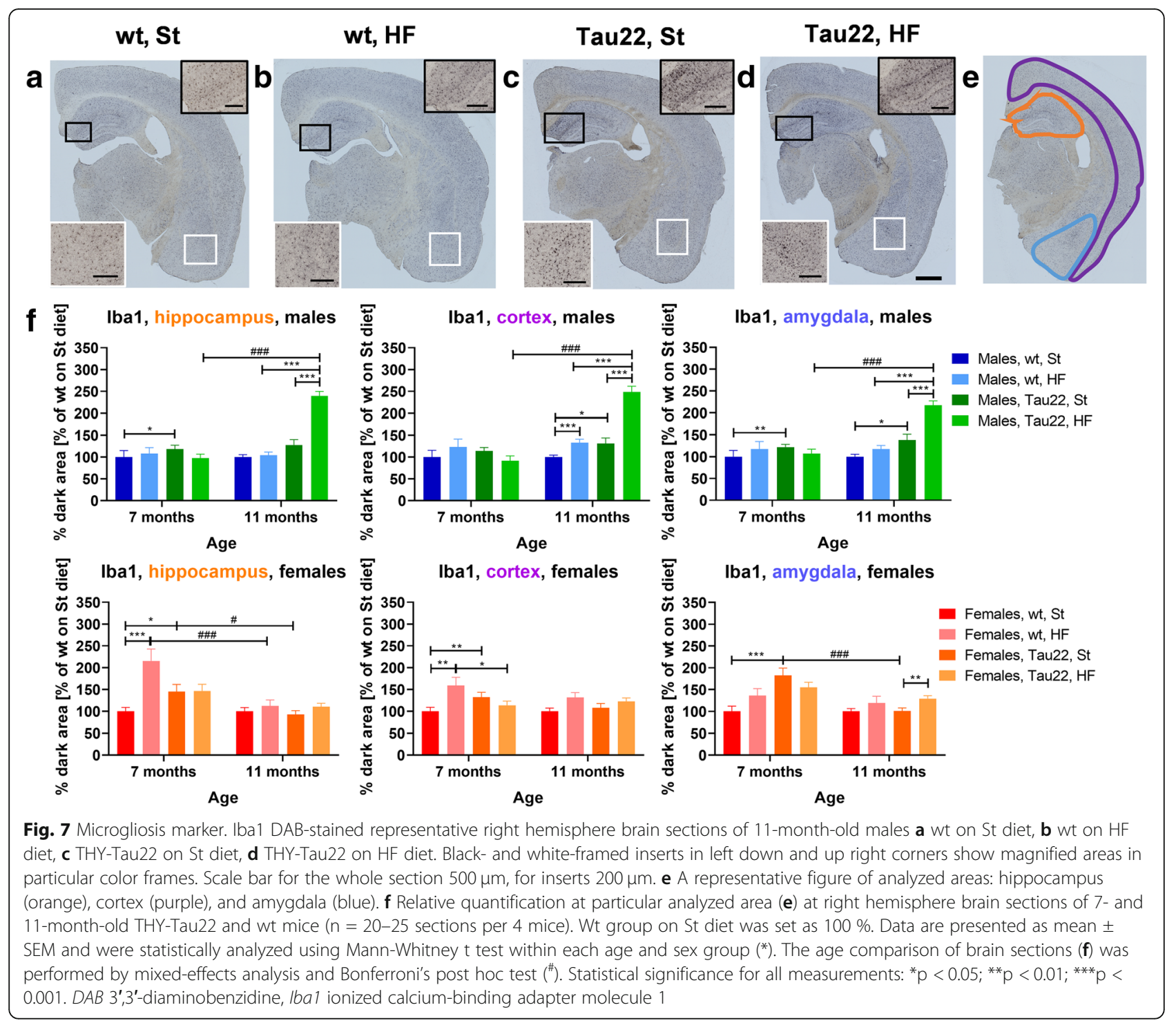

result of IR. However, a previous study by others [16] reported through an insulin tolerance test that 20 weeks of HF-diet feeding did not attenuate peripheral sensitivity to insulin in THY-Tau22 males at 7 months of age. Furthermore, the HF diet-induced obesity led to the higher plasma inflammation demonstrated by an increased level of CRP. Several studies proved the direct link between the amount of body adipocytes which produce proinflammatory cytokines and increased body inflammation in rodents. Such peripheral inflammation could be associated with the central inflammation (reviewed in [2]). However, the present study showed that $\mathrm{C} 57 \mathrm{Bl} / 6 \mathrm{~J}$ mouse controls fed 5 or 9 months of HF diet did not show the direct link between peripheral and central inflammation, neither in males nor in females. A similar situation was observed in THY-Tau22 females, but not males. These results could suggest that in these two particular mouse models, C57Bl/6 J and THY-Tau22, there is no direct link between peripheral and central inflammation, or it could occur only in THY-Tau22 males from the age of 7 months.

Regarding central insulin sensitivity, surprisingly, an enhanced anorexigenic response to centrally administered insulin was reported in THY-Tau22 males 8 to 10 months old with obvious tau pathology and memory deficits [32]. In addition, hippocampal insulin signaling was reported to be increased by the HF diet in 7-monthold THY-Tau22 males [16]. In accordance, 11-monthold THY-Tau22 males fed a St diet showed increased activation of kinases implicated in the insulin signaling cascade as exhibited by increased phosphorylation of PDK-1 at residue S241, Akt at both epitopes T308 and S473, and increased inhibition of GSK-3 $\beta$ kinase activity toward tau protein exhibited by increased 


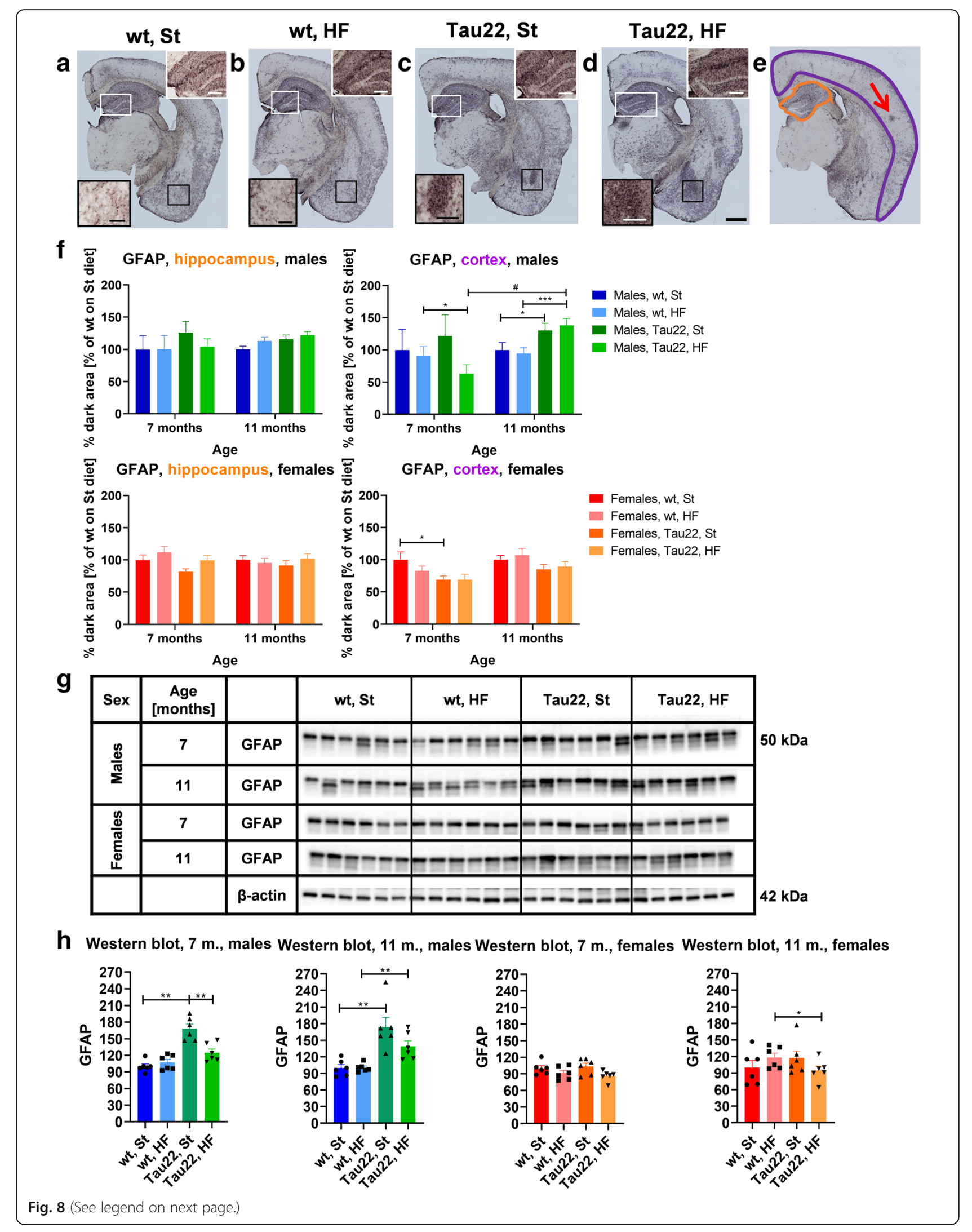


(See figure on previous page.)

Fig. 8 Astrocytosis marker. GFAP DAB-stained representative right hemisphere brain sections of 11-month-old males a wt on St diet, $\mathbf{b}$ wt on HF diet, c THY-Tau22 on St diet, $\mathbf{d}$ THY-Tau22 on HF diet. Black- and white-framed inserts in left down and up right corners show magnified area in particular color frames. Scale bar for the whole section $500 \mu \mathrm{m}$, for inserts $200 \mu \mathrm{m}$. e A representative figure of analyzed areas: hippocampus (orange), cortex (purple). The red arrow point to cluster of reactive astrocytes. $\mathbf{f}$ Relative quantification at particular analyzed area (e) at right hemisphere brain sections of 7- and 11-month-old THY-Tau22 and wt mice ( $\mathrm{n}=24$ sections per 4 mice). Mouse wt group on St diet was set as $100 \%$. $\mathbf{g}$ Western blots of hippocampal GFAP. $\mathbf{h}$ Quantification of $\mathbf{g}$ western blots of hippocampal GFAP in 7- and 11-month-old mice $(\mathrm{n}=6$ ). Mouse wt group on St diet was set as $100 \%$. The intensity of GFAP was related to particular $\beta$-actin intensity. All data are presented as mean \pm SEM and were statistically analyzed using Mann-Whitney $t$ test within each age and sex group $\left(^{*}\right)$. The age comparison of brain sections (F) was performed by mixed-effects analysis and Bonferroni's post hoc test $\left({ }^{*}\right)$. Statistical significance for all measurements: ${ }^{*} p<0.05 ;{ }^{* *} p<0.01 ;{ }^{* * *} p<$

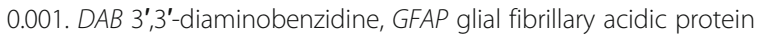

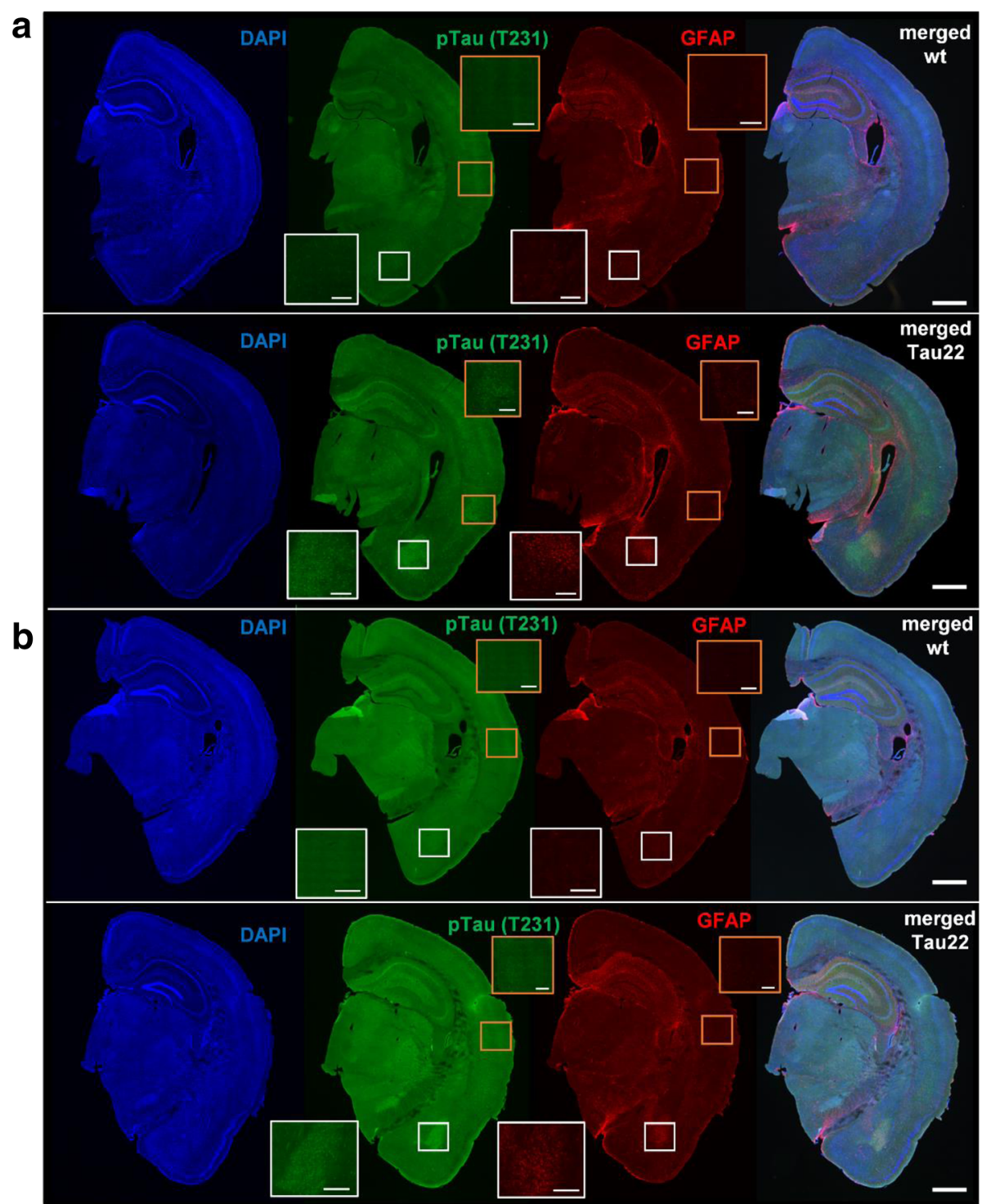

Fig. 9 Fluorescence-labeled THY-Tau22 and wt mouse brains. Colocalization of cell nuclei (blue DAPI), pTau (T231) (green AT180), and activated astrocytes (red GFAP). The representative figures of right hemisphere brain sections of a 7-month-old wt male mouse (upper pictures series) and THY-Tau22 mouse (lower pictures series), both on St diet, and b 11-month-old wt male mouse (upper pictures series) and THY-Tau22 male mouse (lower pictures series), both on HF diet, stained with particular fluorescence antibodies. White- and orange-framed inserts in left down and up right corners show magnified areas in particular color frames. Scale bar for whole brain section $500 \mu \mathrm{m}$, for inserts $200 \mu \mathrm{m}$. GFAP glial fibrillary acidic protein, DAPI 4',6-diamidin-2-fenylindol 


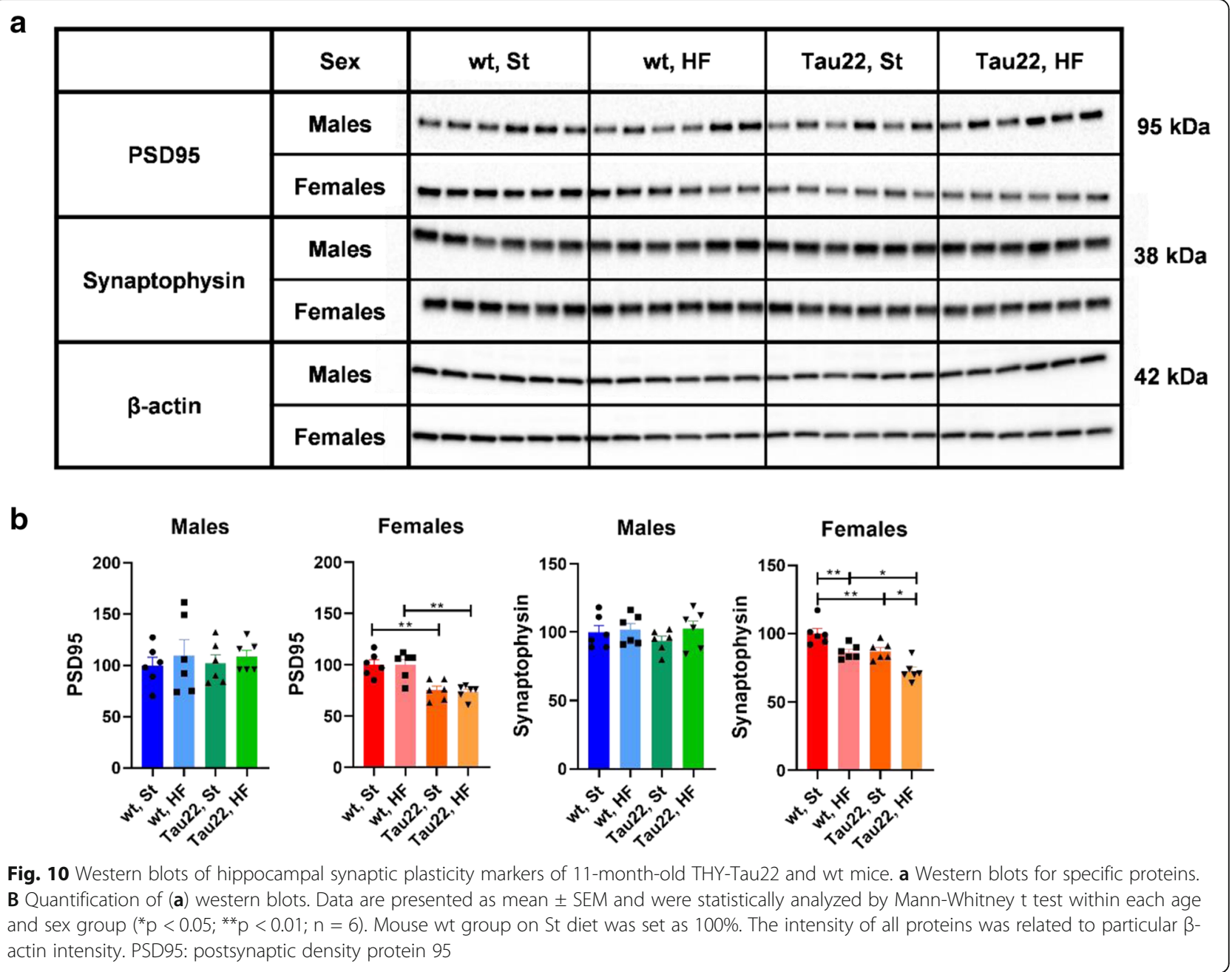

phosphorylation at S9 residue in hippocampi compared to wt males of the same age. However, in our study, 11month-old THY-Tau22 males that were 9 months on the HF diet displayed decreased phosphorylation of pPDK-1 and pAkt at T308, which is directly phosphorylated by $\mathrm{PPDK}-1$. On the other hand, decreased phosphorylation was not observed on Akt at S473; nevertheless, this epitope can be phosphorylated by different kinases such as the mTORC2 complex (mammalian target of rapamycin complex 2) [33]. The impact of the HF diet on the phosphorylation of GSK-3 $\beta$ at S9 residue of 11-month-old THY-Tau22 mice of both sexes was not observed.

However, the activity of another tau kinase, ERK1/2, was significantly increased in 11-month-old THY-Tau22 females fed the HF diet compared to St diet-fed females. The HF diet significantly decreased the inactivated form of PP2A, the main tau phosphatase, pPP2A C (Y307), in THY-Tau22 males. No further impact of the HF diet on PP2A activity was observed.
Overall, the peripheral IR could be defined by increased HOMA and decreased QUICKI indexes. To define the central IR could be more complex and it requires careful interpretation. In this study, we pointed to the differences and changes in mouse hippocampi which could suggest some changes in brain insulin signaling.

In the first study on THY-Tau22 male mice, mild astrocytosis representing brain neuroinflammation was reported [7]. Another study demonstrated brain neuroinflammation in THY-Tau22 mice, shown in the mixture of both sexes, to be triggered by the microglia-originated chemokine CCL3 and to progress with the age of animals [10]. In our study, the progression of microgliosis demonstrated through Iba1 immunohistochemistry was quite different in males and females. In THY-Tau22 males but not females, microgliosis progressed with the age of the animals and was enormously excited by the HF diet. The HF diet increased the amount of clusters of microglia (marked with Iba1) in the hippocampus, cortex, and amygdala of 11-month-old THY-Tau22 males 


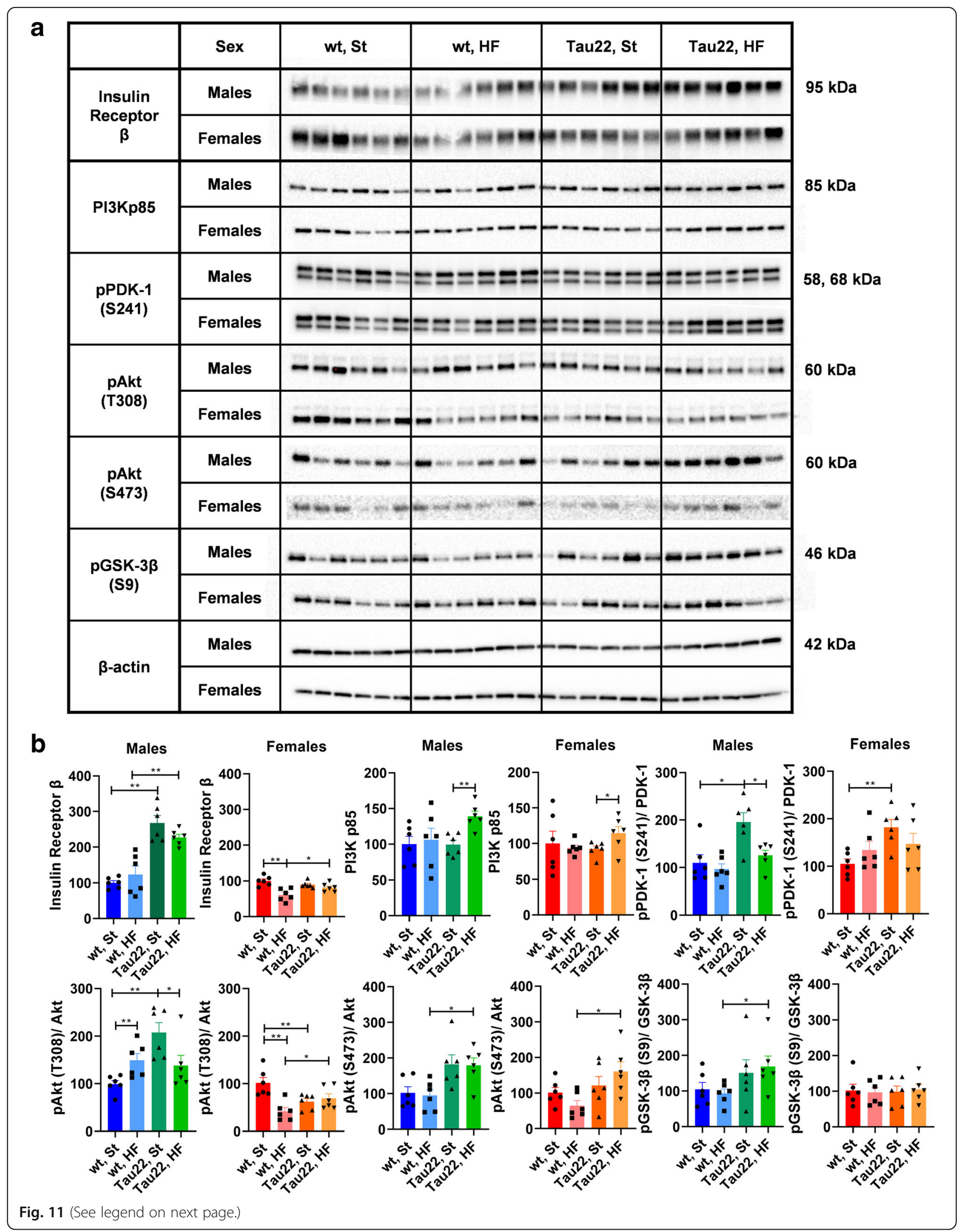


(See figure on previous page.)

Fig. 11 Western blots of hippocampal insulin signaling pathway markers of 11-month-old THY-Tau22 and wt mice. a Western blots for specific proteins. b Quantification of (a) western blots. Data are presented as mean \pm SEM and were statistically analyzed by Mann-Whitney $t$ test within each age and sex group ( ${ }^{*} p<0.05 ;{ }^{* *} p<0.01 ; n=6$ ). Mouse wt group on St diet was set as $100 \%$. The intensity of all proteins was related to particular $\beta$-actin intensity. The intensity of phosphorylated protein was related to the total protein. pAkt, phosphorylated protein kinase B; pPDK1 (S241), phosphoinositide-dependent kinase-1 phosphorylated at S241 residue; pGSK-3 3 , glycogen synthase kinase $3 \beta$ phosphorylated at S9 residue; PI3Kp85, p85 regulatory subunit of phosphoinositide 3-kinase

massively and wt moderately. Previously, HF-diet feeding for 3 months was found to cause an increased incidence of hippocampal Iba1-positive (Iba1+) microglia in 5month-old males of the $\mathrm{C} 57 \mathrm{Bl} / 6 \mathrm{~J}$ strain, which is the basis of THY-Tau22 [34]. A larger effect of 3 months of HF-diet feeding on hippocampal Iba1+ was reported in males but not females in 5-month-old $\mathrm{C} 57 \mathrm{Bl} / 6 \mathrm{~J}$ mice [35]. The WB of hippocampal anti-CD11b antibody showed only one significant increase in 7-month-old transgenic females on the HF diet when compared to wt females on the same diet. This finding could be caused by the fact that the CD11b antigen is expressed on leukocytes in general more than on microglia specifically. Analogously, with an increase in Iba1+ microglia in this study, an enhanced incidence of GFAP-positive (GFAP+) reactive astrocytes was observed in the cortex of 11month-old transgenic males but not females fed the HF diet. This outcome corresponded with the fact that an increase in Iba1+ microglia was observed only in the amygdala of HF diet-fed THY-Tau22 females aged 11 months. This result suggests that neuroinflammation in older THY-Tau22 differed between the sexes and that HF diet-induced obesity led to worsened neuroinflammation in males.

Previous studies showed a higher incidence of activated microglia and reactive astrocytes, both markers of developing neuroinflammation, in close vicinity to the neurofibrillary tangles of hyper and abnormally phosphorylated tau protein (reviewed in [36]). Indeed, our results showed that a high number of reactive astrocytes colocalized with tau protein phosphorylated at the T231 residue in the hippocampus and amygdala in THYTau22 males and females on both the St and HF diets. A lower abundance of colocalized astrocytes and pTau (T231) was also observed in the cortex. The pTau T231 residue was found to engage selectively in a salt bridge with neighboring R230 and to compete for the formation of intermolecular salt bridges to tubulin [37]. Similar results were observed previously in 12-month-old THYTau22 mice of both sexes, where a higher incidence of CD11b-positive $(\mathrm{CD} 11 \mathrm{~b}+)$ and GFAP+ glial cells was reported in the CA1 region of the hippocampus, colocalized with tau phosphorylated at residue S422 [10]. The pTau S422 residue was also suggested to play a role in tau protein aggregation and filament formation [38, 39].
Leboucher et al. showed increased hippocampal tau phosphorylation (S214, S404, and S422), which was due to 20 weeks of HF-diet feeding in 7-month-old THYTau22 males [16]. Even though our study showed increased pTau (T231) colocalized with activated astrocytes by IHC in THY-Tau22 animals when compared to wt animals, the current WB data did not indicate a significant increase in hippocampal tau phosphorylation (at T212, S214, T231, S396, S404, and S422) in THY-Tau22 animals because of HF-diet feeding even at 11 months of age, when the tau pathology was described to be fully developed [7]. However, the increase in pTau colocalized with astrocytes determined by IHC more clearly confirms its pathological effect arising from specific local changes than pTau determined by the WB in a lysate of the whole hippocampus.

In this study, a significant decrease in synaptic and postsynaptic plasticity, expressed by a lower incidence of both synaptophysin and PSD95, was observed only in 11-month-old THY-Tau22 females but not males on both diets compared to wt controls. This result pointed to sex differences in synaptic plasticity of THY-Tau 22 mice and suggested that loss of synaptic plasticity could occur independently of neuroinflammation in THY-Tau22.

The THY-Tau22 mouse model is an artificial model of tau pathology with inserted mutated human tau protein. THY-Tau22 did not evince $A \beta$ pathology and therefore did not fully reflect AD pathology in humans. Therefore, the results from this study should be carefully interpreted when human subjects or other animal model subjects are used in the study. However, a clear sexdependent effect of HF diet-induced obesity on neuroinflammation in mice with mutated human tau was shown. Tau pathology is a main hallmark of human tauopathies, i.e., Pick's disease, and one of the several of main hallmarks of human Alzheimer's disease. Considering the fact that THY-Tau22 mouse model is an artificial model of tau pathology which could influence the overal disease expression, we suggest to consider the sex and overal fitness in age-related human studies related to brain neurodegeneration. Based on our results we propose that 7- to 11-month-old THY-Tau22 males fed both the St and HF diets represent the mouse model of tau pathology with accompanied neuroinflammation suitable for testing of possible therapeutic research. 


\section{a}

\begin{tabular}{|c|c|c|c|}
\hline & Sex & Tau22, St & Tau22, HF \\
\hline \multirow{2}{*}{$\begin{array}{c}\text { pERK1/2 } \\
\text { (T202/Y204) }\end{array}$} & Males & $-\cdots--$ & - - - - \\
\hline & Females & -----1 & $--\cdots-\cdots$ \\
\hline \multirow{2}{*}{ PP2A C } & Males & --- & $-\cdots-\cdots$ \\
\hline & Females & $-\cdots-\cdots$ & $---\cdots$ \\
\hline \multirow{2}{*}{$\begin{array}{c}\text { met-PP2A C } \\
\text { (L309) }\end{array}$} & Males & $---\cdots$ & ------ \\
\hline & Females & $---\cdots-$ & $----\cdots$ \\
\hline \multirow{2}{*}{$\begin{array}{l}\text { pPP2A } \\
\text { (Y307) }\end{array}$} & Males & $-\cdots-\cdots$ & $-1-\cdots$ \\
\hline & Females & $-\cdots-\cdots$ & $-\cdots-\cdots$ \\
\hline \multirow{2}{*}{$\beta$-actin } & Males & $-\cdots-\cdots$ & $-\cdots-\cdots$ \\
\hline & Females & $-\cdots-\cdots$ & $-\cdots \cdots$ \\
\hline
\end{tabular}

b

Males

Females

Males

Females
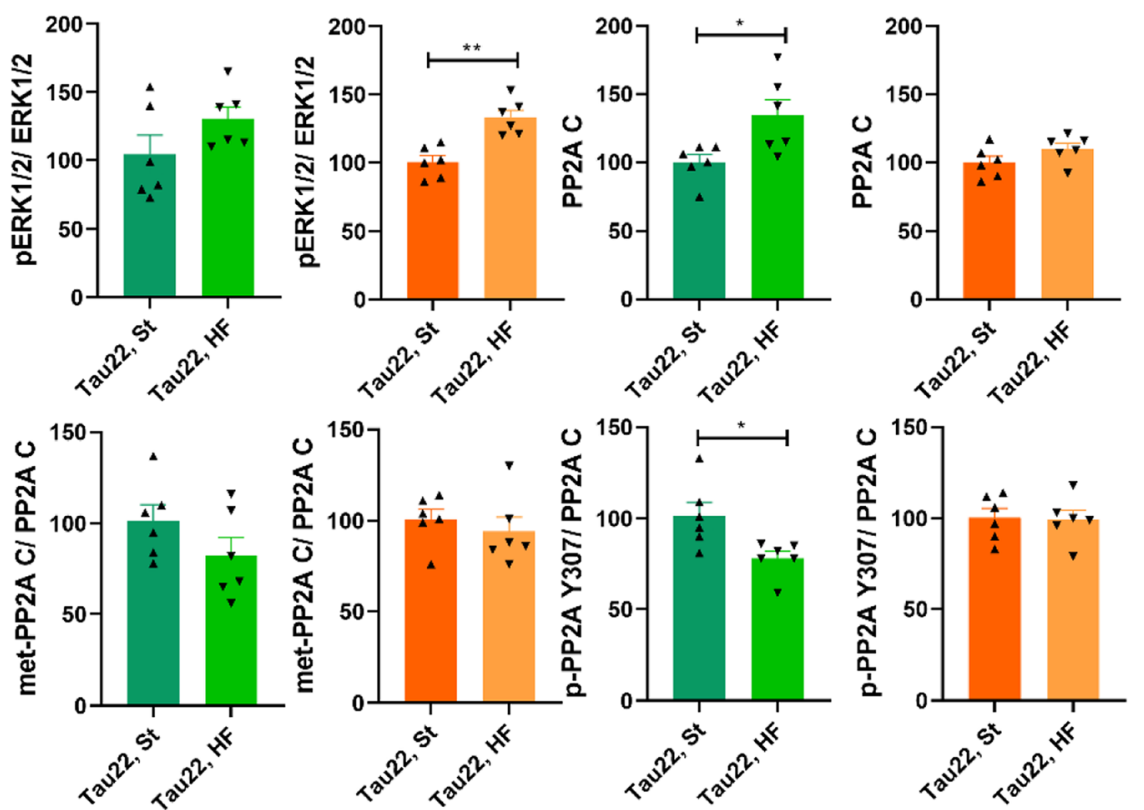

Fig. 12 Western blots of hippocampal tau protein de/phosphorylation enzymes of 11-month-old THY-Tau22 mice. a Western blots for specific proteins. b Quantification of (a) western blots. Data are presented as mean \pm SEM and were statistically analyzed by Mann-Whitney t test within each age and sex group ( $\left.{ }^{*} p<0.05 ;{ }^{* *} p<0.01 ; n=6\right)$. Mouse THY-Tau22 group on St diet was set as $100 \%$. The intensity of all proteins was related to particular $\beta$-actin intensity. The intensity of modified protein was related to the total protein. pERK1/2, extracellular signal-regulated kinase phosphorylated at T202/Y204 residues; PP2A C, C subunit of protein phosphatase 2A; met-PP2A C (L309), methylated C subunit of PP2A at L309 residue; PPP2A C (Y307), PP2A C phosphorylated at Y307 residue

Furthermore, 11-month-old THY-Tau22 females fed both the St and HF diets represent the mouse model of tau pathology with accompanied worsened synapthogenesis suitable for testing of possible therapeutic research.

\section{Conclusions}

To conclude, the presented results showed strong neuroinflammation represented by microgliosis (marked by Iba1) and astrocytosis (marked by GFAP) in 11-month- 
Table 9 Direct activity of kinases/ PP2A phosphatase on ADpotentially de/phosphorylated tau residues relevant for this study [25-29]

\begin{tabular}{llll}
\hline Phosphorylated tau residue & ERK1/2 & GSK-3 $\beta$ & PP2A \\
\hline T212 & $\checkmark$ & $\checkmark$ & $\checkmark$ \\
S214 & & $\checkmark$ & $\checkmark$ \\
T231 & $\checkmark$ & $\checkmark$ & \\
S396 & $\checkmark$ & $\checkmark$ & $\checkmark$ \\
S404 & $\checkmark$ & $\checkmark$ & $\checkmark$ \\
S422 & $\checkmark$ & & \\
\hline
\end{tabular}

ERK1/2 extracellular signal-regulated kinase $1 / 2$ known as mitogen-activated protein kinase MAPK, GSK-3 $\beta$ glycogen synthase kinase 3 beta; PP2A: protein phosphatase $2 \mathrm{~A}$ old THY-Tau22 males but not females. Such strong neuroinflammation was accompanied by massive tau protein phosphorylation at the T231 residue, which was mostly visible in the hippocampus and amygdala and moderately visible in the cortex. Furthermore, probably resulting from increased neuroinflammation in the brains of THY-Tau22 males, but not females, these males evinced impaired short-term spatial memory. Moreover, 11month-old females, but not males, showed robustly worsened synaptic and postsynaptic plasticity even though they did not show worsened neuroinflammation caused by the tau transgene or HF diet. Such results indicated significant differences between the sexes of
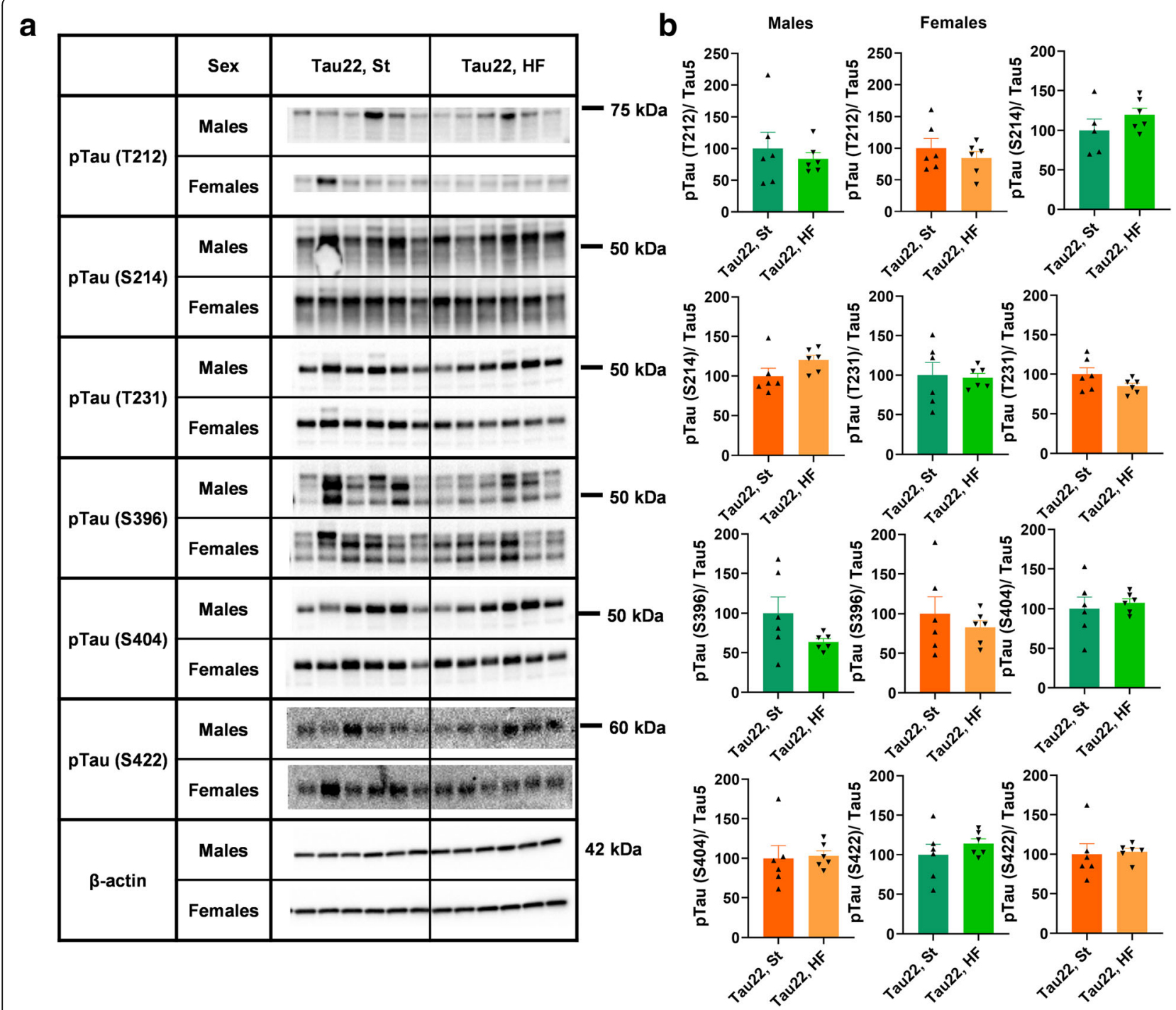

Fig. 13 Western blots of hippocampal phosphorylated tau proteins of 11-month-old THY-Tau22 mice. a Western blots for specific proteins. $\mathbf{b}$ Quantification of (a) western blots. Data are presented as mean \pm SEM and were statistically analyzed using Mann-Whitney $t$ test within each age and sex group. No statistically significant difference was observed. ( $p \geq 0.05 ; n=5-6$ ). Mouse THY-Tau22 group on St diet was set as $100 \%$. The intensity of phosphorylated tau proteins was related to the Tau 5 total protein 
THY-Tau22 mice, and this finding should be kept in mind in future studies.

The HF diet caused a significant increase in body weight and related metabolic parameters, which led to peripheral IR in both transgenic and wt mice of both sexes. The present study linked the HF diet not only to peripheral IR but also to worsened neuroinflammation in the blood plasma of all HF-diet fed mice, and in the male THY-Tau22 brain.

\begin{abstract}
Abbreviations
AB: Beta-amyloid; AD: Alzheimer's disease; Akt: Protein kinase B; pAkt: Akt phosphorylated at T308 and/or S473 residues; ANOVA: Analysis of variance; $A \cup C_{\triangle G \text { Glucose: }}$ Area under the curve calculated from OGTT;

BIOCEV: Biotechnology and Biomedicine Centre of the Academy of Sciences and Charles University; BSA: Bovine serum albumin; CD1 1b: Cluster of differentiation molecule 11b known as integrin alpha M; CRP: C-reactive protein; DAB: 3,3'-diaminobenzidine; DAPI: 4',6'-diamidin-2-phenylindole; ERK1/2: Extracellular signal-regulated kinase 1/2 known as mitogen-activated protein kinase, MAPK; pERK1/2 (T202/Y204): ERK1/2 phosphorylated at T202/ Y204 residues; FFA: Free fatty acids; GFAP: Glial fibrillary acidic protein;

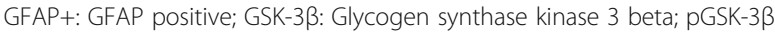
(S9): GSK-33 phosphorylated at S9 residue; HF: High-fat diet; HOMAIR: Homeostatic model assessment of insulin resistance; Iba1: Ionized calcium-binding adaptor molecule 1; Iba1+: Iba1 positive; IPAT: Intraperitoneal adipose tissue; IR: Insulin resistance; mAb: Monoclonal antibody; NZ: New zone in Y-maze test; NGS: Normal goat serum; OGTT: Oral glucose tolerance test; pAb: Polyclonal antibody; PDK-1: Phosphoinositidedependent kinase-1; PPDK-1 (S241): PDK-1 phosphorylated at S241 residue; PI3Kp85: p85 regulatory subunit of phosphoinositide 3-kinase; PP2A: Protein phosphatase 2A; met-PP2A C (L309): PP2A C subunit methylated at L309 residue; PPP2A C (Y307): Phosphorylated PP2A C subunit at Y307 residue; QUICKI: Quantitative insulin sensitivity check index; PSD95: Postsynaptic density protein 95; SCAT: Subcutaneous adipose tissue; SEM: Standard error of mean; St: Standard diet; pTau: Phosphorylated tau protein; Tau22 and THY-Tau22: THY-Tau22 genotype; TG: Triglyceride; W: Week; WB: Western blots; wt: Wild type
\end{abstract}

\section{Supplementary Information}

The online version contains supplementary material available at https://doi. org/10.1186/s12974-021-02190-3.

Additional file 1: Fig. S1. Behavioral test. Open field $(n=7-14)$. Data are presented as mean \pm SEM and were statistically analyzed by MannWhitney t-test $\left(^{*}\right)$ within each age and sex group $\left({ }^{*} p<0.05 ;{ }^{* *} p<0.01\right)$. The age comparison was performed by mixed-effects analysis and Bonferroni's post hoc test $(\#)$. A value significance between 11 and 3 , and 11 and 7 months in particular group at wall distance was ${ }^{\#} p<0.001$ in all cases (not shown for clarity). Fig. S2 Western blots of hippocampal microgliosis marker CD11b. (A) Western blots for specific proteins. (B) Quantification of (A) western blots. Data are presented as mean \pm SEM and were statistically analyzed by Mann-Whitney t-test within each age and sex group ( $\left.{ }^{*} p<0.05 ; n=6\right)$. Mouse wt group on $\mathrm{St}$ diet was set as $100 \%$. The intensity of all proteins was related to particular $\beta$-actin intensity. CD11b: cluster of differentiation molecule $11 \mathrm{~b}$ known as integrin alpha M; Iba1: ionized calcium-binding adaptor molecule 1. Fig. S3 Western blots of hippocampal insulin signaling pathway markers of 11-monthold THY-Tau22 and wt mice. (A) Western blots for specific proteins. (B) Quantification of (A) western blots. Data are presented as mean \pm SEM and were statistically analyzed by Mann-Whitney t-test within each age and sex group $\left({ }^{*} p<0.05 ;{ }^{* *} p<0.01 ; n=6\right)$. Mouse wt group on St diet was set as $100 \%$. The intensity of all proteins was related to particular $\beta$ actin intensity. Akt: protein kinase B; GSK-3B: glycogen synthase kinase 3 beta; PDK-1: phosphoinositide-dependent kinase-1. Fig. S4 Western blots of hippocampal ERK1/2 of 11-month-old THY-Tau22 and wt mice. (A) Western blots for specific proteins. (B) Quantification of (A) western blots. Data are presented as mean \pm SEM and were statistically analyzed by
Mann-Whitney t-test within each age and sex group $\left({ }^{* *} \mathrm{p}<0.01 ; \mathrm{n}=6\right.$ ) Mouse THY-Tau22 group on St diet was set as $100 \%$. The intensity of all proteins was related to particular $\beta$-actin intensity. ERK1/2: extracellular signal regulated kinase. Fig. $\mathbf{S 5}$ Western blots of hippocampal Tau5 of 11-month-old THY-Tau22 mice. (A) Western blots for specific proteins. (B) Quantification of (A) western blots. Data are presented as mean \pm SEM and were statistically analyzed using Mann-Whitney t-test. No statistically significant difference was observed. $(p \geq 0.05 ; n=6)$. Mouse THY-Tau22 group on St diet was set as $100 \%$. The intensity of Tau 5 was related to particular $\beta$-actin intensity.

\section{Acknowledgements}

Results of this study were obtained using the infrastructure of the Czech Centre for Phenogenomics supported by projects of the Ministry of Education, Youth, and Sports and European Regional Development Fund (ERDF) LM2018126, OP VaVpl CZ.1.05/2.1.00/19.0395 and CZ.1.05/1.1.00/ 02.0109. We would like to thank Martina Kojecká and Lucia Kořínková for biochemical parameters measurements, and for liver histology, Hedvika Vysušilová for her excellent technical assistance and animal care, Marta Sýkorová and Eliška Motková for performing of behavioral experiments, and Marie-Christine Galas for the manuscript revision.

\section{Authors' contributions}

L.M. supervised the project. L.M. and M.H. designed the study. M.K. and M.B. performed western blots and immunohistochemistry experiments. M.K. performed behavioral tests and OGT, analyzed the data. B.S. performed all statistical analyses. M.K. and B.Ž. wrote manuscript. M.K., B.Ž., J.K., A.P., and L.M. discussed the results. All authors read and commented on the final version of the manuscript. The author(s) read and approved the final manuscript.

\section{Funding}

This study was supported by Czech Science Foundation [grant number 20-00546S] and by the Academy of Sciences of the Czech Republic [grant numbers RVO: 61388963 and RVO: 67985823]

\section{Availability of data and materials}

The datasets generated during and/or analyzed during the current study are available from the corresponding author on reasonable request. The supplementary file including figures supporting this study is included in this published article.

\section{Declarations}

\section{Ethics approval and consent to participate}

The animal experiments followed the ethical guidelines for animal experiments in the Czech Republic Act Nr. 246/1992 and were approved by the Committee for Experiments with Laboratory Animals of the Czech Academy of Sciences.

\section{Consent for publication}

Not applicable.

\section{Competing interests}

The authors declare that there are no competing interests associated with the manuscript.

\section{Author details}

${ }^{1}$ Institute of Organic Chemistry and Biochemistry of the Czech Academy of Sciences, Flemingovo náměstí 542/2, 16000 Prague 6, Czech Republic. ${ }^{2}$ Institute of Physiology of the Czech Academy of Sciences, Vídeňská 1083, 14220 Prague 4, Czech Republic. ${ }^{3}$ Department of Mathematics, University of West Bohemia, Univerzitní 2732/8, 30100 Pilsen, Czech Republic.

Received: 12 March 2021 Accepted: 1 June 2021

Published online: 22 June 2021

\section{References}

1. Bray GA. Medical consequences of obesity. J Clin Endocrinol Metab. 2004; 89(6):2583-9. https://doi.org/10.1210/jc.2004-0535. 
2. Kacirova M, Zmeskalova A, Korinkova L, Zelezna B, Kunes J, Maletinska L. Inflammation: major denominator of obesity, Type 2 diabetes and Alzheimer's disease-like pathology? Clin Sci (Lond). 2020;134(5):547-70. https://doi.org/10.1042/CS20191313.

3. Guillemot-Legris O, Muccioli GG. Obesity-Induced Neuroinflammation: Beyond the Hypothalamus. Trends Neurosci. 2017;40(4):237-53. https://doi. org/10.1016/j.tins.2017.02.005

4. Korinkova L, Holubova M, Neprasova B, Hruba L, Prazienkova V, Bencze M, et al. Synergistic effect of leptin and lipidized PrRP on metabolic pathways in ob/ob mice. J Mol Endocrinol. 2020;64(2):77-90. https://doi.org/10.1530/ JME-19-0188.

5. Alford S, Patel D, Perakakis N, Mantzoros CS. Obesity as a risk factor for Alzheimer's disease: weighing the evidence. Obes Rev. 2018;19(2):269-80. https://doi.org/10.1111/obr.12629

6. Grundke-labal I, labal K, Tung YC, Quinlan M, Wisniewski HM, Binder LI. Abnormal phosphorylation of the microtubule-associated protein tau (tau) in Alzheimer cytoskeletal pathology. Proc Natl Acad Sci U S A. 1986;83(13): 4913-7. https://doi.org/10.1073/pnas.83.13.4913.

7. Schindowski K, Bretteville A, Leroy K, Begard S, Brion JP, Hamdane M, et al. Alzheimer's disease-like tau neuropathology leads to memory deficits and loss of functional synapses in a novel mutated tau transgenic mouse without any motor deficits. Am J Pathol. 2006;169(2):599-616. https://doi. org/10.2353/ajpath.2006.060002.

8. Belarbi K, Burnouf S, Fernandez-Gomez FJ, Desmercieres J, Troquier L, Brouillette J, et al. Loss of medial septum cholinergic neurons in THY-Tau22 mouse model: what links with tau pathology? Curr Alzheimer Res. 2011;8(6): 633-8. https://doi.org/10.2174/156720511796717230.

9. Van der Jeugd A, Vermaercke B, Derisbourg M, Lo AC, Hamdane M, Blum D, et al. Progressive age-related cognitive decline in tau mice. J Alzheimers Dis. 2013;37(4):777-88. https://doi.org/10.3233/JAD-130110.

10. Laurent C, Dorothee G, Hunot S, Martin E, Monnet Y, Duchamp M, et al. Hippocampal T cell infiltration promotes neuroinflammation and cognitive decline in a mouse model of tauopathy. Brain. 2017;140(1):184-200. https:// doi.org/10.1093/brain/aww270.

11. Luo Y, Kaur C, Ling EA. Neuronal and glial response in the rat hypothalamus-neurohypophysis complex with streptozotocin-induced diabetes. Brain Res. 2002;925(1):42-54. https://doi.org/10.1016/S0006-8993 (01)03258-9.

12. Thaler JP, Yi CX, Schur EA, Guyenet SJ, Hwang BH, Dietrich MO, et al. Obesity is associated with hypothalamic injury in rodents and humans. J Clin Invest. 2012;122(1):153-62. https://doi.org/10.1172/JCI59660.

13. Becker K, Freude S, Zemva J, Stohr O, Krone W, Schubert M. Chronic peripheral hyperinsulinemia has no substantial influence on tau phosphorylation in vivo. Neurosci Lett. 2012;516(2):306-10. https://doi. org/10.1016/j.neulet.2012.04.022.

14. Moroz N, Tong M, Longato L, Xu H, de la Monte SM. Limited Alzheimertype neurodegeneration in experimental obesity and type 2 diabetes mellitus. J Alzheimers Dis. 2008;15(1):29-44. https://doi.org/10.3233/JAD-2 008-15103.

15. van der Harg JM, Eggels L, Ruigrok SR, Hoozemans JJ, la Fleur SE, Scheper W. Neuroinflammation is not a Prerequisite for Diabetes-induced Tau Phosphorylation. Front Neurosci. 2015;9:432.

16. Leboucher A, Laurent C, Fernandez-Gomez FJ, Burnouf S, Troquier L, Eddarkaoui S, et al. Detrimental effects of diet-induced obesity on tau pathology are independent of insulin resistance in tau transgenic mice. Diabetes. 2013;62(5):1681-8. https://doi.org/10.2337/db12-0866.

17. Mangold CA, Wronowski B, Du M, Masser DR, Hadad N, Bixler GV, et al. Sexually divergent induction of microglial-associated neuroinflammation with hippocampal aging. J Neuroinflammation. 2017;14(1):141. https://doi. org/10.1186/s12974-017-0920-8

18. Prazienkova V, Holubova M, Pelantova H, Buganova M, Pirnik Z, Mikulaskova $B$, et al. Impact of novel palmitoylated prolactin-releasing peptide analogs on metabolic changes in mice with diet-induced obesity. PLoS One. 2017; 12(8):e0183449. https://doi.org/10.1371/journal.pone.0183449.

19. Seibenhener ML, Wooten MC. Use of the Open Field Maze to measure locomotor and anxiety-like behavior in mice. J Vis Exp. 2015;96:e52434

20. Popelova A, Prazienkova V, Neprasova B, Kasperova BJ, Hruba L, Holubova $M$, et al. Novel Lipidized Analog of Prolactin-Releasing Peptide Improves Memory Impairment and Attenuates Hyperphosphorylation of Tau Protein in a Mouse Model of Tauopathy. J Alzheimers Dis. 2018;62(4):1725-36. https://doi.org/10.3233/JAD-171041.
21. Pelantova H, Buganova M, Holubova M, Sediva B, Zemenova J, Sykora D, et al. Urinary metabolomic profiling in mice with diet-induced obesity and type 2 diabetes mellitus after treatment with metformin, vildagliptin and their combination. Mol Cell Endocrinol. 2016;431:88-100. https://doi.org/10.1 016/j.mce.2016.05.003.

22. Holubova M, Hruba L, Popelova A, Bencze M, Prazienkova V, Gengler S, et al. Liraglutide and a lipidized analog of prolactin-releasing peptide show neuroprotective effects in a mouse model of beta-amyloid pathology. Neuropharmacology. 2019;144:377-87. https://doi.org/10.1016/j.neuropha rm.2018.11.002.

23. Matyskova R, Maletinska L, Maixnerova J, Pirnik Z, Kiss A, Zelezna B. Comparison of the obesity phenotypes related to monosodium glutamate effect on arcuate nucleus and/or the high fat diet feeding in C57BL/6 and NMRI mice. Physiol Res. 2008;57(5):727-34. https://doi.org/10.33549/physiolres.931274.

24. Spolcova A, Mikulaskova B, Krskova K, Gajdosechova L, Zorad S, Olszanecki $\mathrm{R}$, et al. Deficient hippocampal insulin signaling and augmented Tau phosphorylation is related to obesity- and age-induced peripheral insulin resistance: a study in Zucker rats. BMC Neurosci. 2014;15(1):111. https://doi. org/10.1186/1471-2202-15-111.

25. Hanger DP, Anderton BH, Noble W. Tau phosphorylation: the therapeutic challenge for neurodegenerative disease. Trends Mol Med. 2009;15(3):112-9. https://doi.org/10.1016/j.molmed.2009.01.003.

26. Ksiezak-Reding H, Pyo HK, Feinstein B, Pasinetti GM. Akt/PKB kinase phosphorylates separately Thr212 and Ser214 of tau protein in vitro. Biochim Biophys Acta. 2003;1639(3):159-68. https://doi.org/10.1016/j.bbadis.2003.09.001.

27. Qi H, Prabakaran S, Cantrelle FX, Chambraud B, Gunawardena J, Lippens G, et al. Characterization of Neuronal Tau Protein as a Target of Extracellular Signal-regulated Kinase. J Biol Chem. 2016;291(14):7742-53. https://doi.org/1 $0.1074 / j b c . M 115.700914$

28. Reynolds $\mathrm{CH}$, Betts JC, Blackstock WP, Nebreda AR, Anderton BH. Phosphorylation sites on tau identified by nanoelectrospray mass spectrometry: differences in vitro between the mitogen-activated protein kinases ERK2, c-Jun N-terminal kinase and P38, and glycogen synthase kinase-3beta. J Neurochem. 2000;74(4):1587-95. https://doi.org/10.1046/j.1471-4159.2000.0741587.x.

29. Utton MA, Vandecandelaere A, Wagner U, Reynolds CH, Gibb GM, Miller CC, et al. Phosphorylation of tau by glycogen synthase kinase 3 beta affects the ability of tau to promote microtubule self-assembly. Biochem J. 1997;323(Pt 3):741-7. https://doi.org/10.1042/bj3230741.

30. Ebbert JO, Jensen MD. Fat depots, free fatty acids, and dyslipidemia. Nutrients. 2013;5(2):498-508. https://doi.org/10.3390/nu5020498.

31. Maletinska L, Nagelova $V$, Ticha A, Zemenova J, Pirnik Z, Holubova M, et al. Novel lipidized analogs of prolactin-releasing peptide have prolonged halflives and exert anti-obesity effects after peripheral administration. Int J Obes (Lond). 2015;39(6):986-93. https://doi.org/10.1038/ijo.2015.28.

32. Leboucher A, Ahmed T, Caron E, Tailleux A, Raison S, Joly-Amado A, et al. Brain insulin response and peripheral metabolic changes in a Tau transgenic mouse model. Neurobiol Dis. 2019;125:14-22. https://doi.org/10.1016/j.nbd.2019.01.008.

33. Gao Y, Moten A, Lin HK. Akt: a new activation mechanism. Cell Res. 2014; 24(7):785-6. https://doi.org/10.1038/cr.2014.57.

34. Hao S, Dey A, Yu X, Stranahan AM. Dietary obesity reversibly induces synaptic stripping by microglia and impairs hippocampal plasticity. Brain Behav Immun. 2016;51:230-9. https://doi.org/10.1016/j.bbi.2015.08.023.

35. Robison LS, Albert NM, Camargo LA, Anderson BM, Salinero AE, Riccio DA, et al. High-Fat Diet-Induced Obesity Causes Sex-Specific Deficits in Adult Hippocampal Neurogenesis in Mice. eNeuro. 2020;7(1):1-17.

36. Ferrer I. Astrogliopathy in tauopathies. Neuroglia. 2018;1(1):126-50. https:// doi.org/10.3390/neuroglia1010010.

37. Schwalbe M, Kadavath H, Biernat J, Ozenne V, Blackledge M, Mandelkow E, et al. Structural Impact of Tau Phosphorylation at Threonine 231. Structure. 2015;23(8):1448-58. https://doi.org/10.1016/j.str.2015.06.002.

38. Ferrari A, Hoerndli F, Baechi T, Nitsch RM, Gotz J. beta-Amyloid induces paired helical filament-like tau filaments in tissue culture. J Biol Chem. 2003; 278(41):40162-8. https://doi.org/10.1074/jbc.M308243200.

39. Stoothoff WH, Johnson GV. Tau phosphorylation: physiological and pathological consequences. Biochim Biophys Acta. 2005;1739(2-3):280-97. https://doi.org/10.1016/j.bbadis.2004.06.017.

\section{Publisher's Note}

Springer Nature remains neutral with regard to jurisdictional claims in published maps and institutional affiliations. 